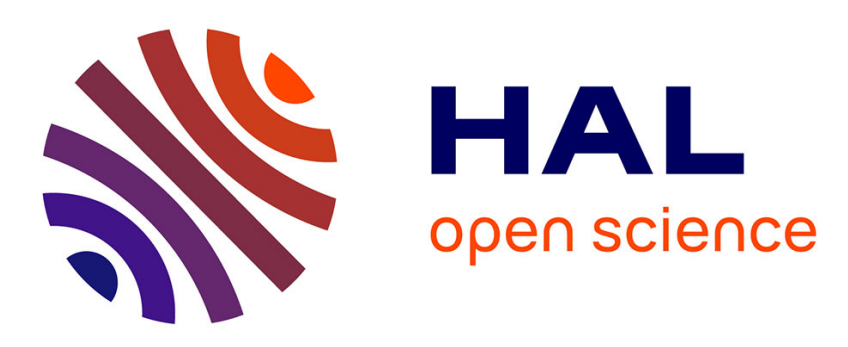

\title{
Intramolecular and intermolecular dynamics in molecular liquids through femtosecond time-resolved impulsive stimulated scattering
}

S. Ruhman, A.G. Joly, B. Kohler, L.R. Williams, K.A. Nelson

\section{To cite this version:}

S. Ruhman, A.G. Joly, B. Kohler, L.R. Williams, K.A. Nelson. Intramolecular and intermolecular dynamics in molecular liquids through femtosecond time-resolved impulsive stimulated scattering. Revue de Physique Appliquée, 1987, 22 (12), pp.1717-1734. 10.1051/rphysap:0198700220120171700 . jpa-00245732

\section{HAL Id: jpa-00245732 \\ https://hal.science/jpa-00245732}

Submitted on 1 Jan 1987

HAL is a multi-disciplinary open access archive for the deposit and dissemination of scientific research documents, whether they are published or not. The documents may come from teaching and research institutions in France or abroad, or from public or private research centers.
L'archive ouverte pluridisciplinaire HAL, est destinée au dépôt et à la diffusion de documents scientifiques de niveau recherche, publiés ou non, émanant des établissements d'enseignement et de recherche français ou étrangers, des laboratoires publics ou privés. 


\title{
Intramolecular and intermolecular dynamics in molecular liquids through femtosecond time-resolved impulsive stimulated scattering
}

\author{
S. Ruhman (*), A. G. Joly, B. Kohler, L. R. Williams (**) and K. A. Nelson (***) \\ Department of Chemistry, Massachusetts Institute of Technology, Cambridge, Massachusetts 02139, U.S.A.
}

(Reçu le 10 juillet 1987, révisé le 21 septembre 1987, accepté le 21 septembre 1987)

\begin{abstract}
Résumé. - Des résultats expérimentaux récents sur la diffusion stimulée impulsionnelle (ISS) résolue aux temps femtosecondes dans les liquides moléculaires sont passés en revue et de nouvelles données sont présentées. Trois points sont discutés. En premier, les mouvements moléculaires inertiels de liquides simples $\left(\mathrm{CS}_{2}\right.$, benzène) sont observés. Ces résultats peuvent être interprétés en termes de librations intermoléculaires, qui dans quelques cas se manifestent nettement par des réponses oscillatoires dépendant du temps. La fréquence de libration intermoléculaire dépendant de la température et la dynamique du déphasage dans le $\mathrm{CS}_{2}$ liquide sont déterminées. Les valeurs suggèrent que le déphasage est principalement inhomogène et elles permettent d'estimer le degré d'inhomogénéité du potentiel de libration. Deuxièmement, les oscillations vibrationnelles intramoléculaires sont suivies directement en temps réel. Les valeurs sont influencées à la fois par la dynamique moléculaire de vibration et par celle d'orientation. Ces expériences rendent possible la spectroscopie résolue en temps des molécules vibrationnellement distordues dans des configurations non équilibrées bien définies. Finalement, on discute l'apparition généralisée de diffusion stimulée impulsionnelle chaque fois qu'une impulsion ultracourte de lumière traverse un milieu actif en Raman.
\end{abstract}

\begin{abstract}
Recent femtosecond time-resolved impulsive stimulated scattering experimental results from molecular liquids are reviewed and new data are presented. Three main areas are discussed. First, the inertial molecular motions of simple liquids ( $\mathrm{CS}_{2}$, benzene) are observed. The results can be interpreted in terms of intermolecular librations, which in some cases are clearly manifest in the form of oscillatory time-dependent responses. The temperature-dependent intermolecular librational frequency and dephasing dynamics in $\mathrm{CS}_{2}$ liquid are determined. The data suggest that dephasing is predominantly inhomogeneous, and permit estimation of the extent of inhomogeneity in the librational frequency. Second, intramolecular vibrational oscillations are monitored directly in real time. The data are influenceed by both molecular vibrational and orientational dynamics. These experiments make possible time-resolved spectroscopy of vibrationally distorted molecules in well defined nonequilibrium configurations. Finally, the general occurrence of impulsive stimulated scattering whenever an ultrashort light pulse passes through a Raman-active medium is discussed.
\end{abstract}

\section{Introduction.}

The dynamical behaviour of molecular liquids has been a subject of extensive theoretical and experimental study [1-4]. Intramolecular vibrations and their dissipation and dephasing mechanisms, intermolecular «collisions », and molecular orientational motions have all been explored. Among the experimental techniques brought to bear on this area, spontaneous frequency-resolved light scattering spectroscopy has been used most [1-7]. Raman

$\left(^{*}\right)$ Weizmann Postdoctoral Fellow.

(**) AT \& T Bell Laboratories Ph. D. Scholar.

$(* * *)$ Presidential Young Investigator Awardee and Alfred P. Sloan Fellow. spectra of molecular vibrational modes contain information about the vibrational frequencies and dephasing rates as well as the dynamics of molecular orientational motions. Brillouin spectra yield frequencies and attenuation rates of longitudinal acoustic waves and, in some viscoelastic fluids, shear acoustic waves. Rayleigh spectral profiles contain information about collective orientational motion and local intermolecular «collisions » or vibrations.

Recently, an entirely time-domain form of light scattering spectroscopy has been developed and applied to the study of simple and viscoelastic molecular liquids $[8,9]$. «Impulsive " stimulated light scattering (ISS) permits extraction of the full information content of Raman, Brillouin, and 
Rayleigh spectral features in the time domain. ISS has proved to be complementary to frequencydomain light scattering (LS) in several cases where the latter may present difficulties, e.g. in the study of low-frequency acoustic modes, in the use of small scattering angles for characterization of dispersive rodes, in some coupled-mode systems, and in the study of vibrational modes which undergo very rapid dissipation or dephasing [9-11].

In this paper we review several recent femtosecond time-resolved ISS investigations of molecular liquids and present some new results. Two areas of investigation will be discussed. The first deals primarily with intermolecular vibrational motion which can be observed directly on the $0-1$ ps time scale in many liquids [8, 12-17]. This type of motion influences the wings of the quasielastic LS spectrum, but due to very rapid vibrational dephasing its characterization is extremely difficult in the frequency domain [1-7, 17-24]. Our results facilitate determination of intermolecular librational frequencies and dephasing dynamics. In carbon disulphide $\left(\mathrm{CS}_{2}\right)$ liquid, temperature-dependent results suggest that very rapid intermolecular vibrational dephasing is due primarily to inhomogeneity in local librational frequencies, and permit estimation of the extent of inhomogeneity $[13,14]$.

We also discuss very recent impulsive stimulated Raman scattering (ISRS) experiments in which direct femtosecond time-resolved observations of intramolecular vibrational oscillations have been made $[8,25,26]$. These experiments, analogous to spontaneous Raman scattering, can provide information about molecular vibrational frequencies and dephasing dynamics, and also about single-molecule orientational correlations [1-6, 20, 27-34]. Most importantly, they permit various types of time-resolved spectroscopy to be carried out on well defined, vibrationally distorted (e.g. stretched or bent) molecules.

The experiments on molecular vibrations have also demonstrated the very general occurrence of impulsive stimulated Raman scattering [8, 25, 26]. As was predicted theoretically [35], ISRS must occur (with no laser intensity threshold) whenever a sufficiently short light pulse passes through any Raman active medium. This results in coherent excitation of the medium as well as changes in the spectral content of the ultrashort pulse. The general importance of ISS (and other « impulsive » interactions) in ultrafast optics and spectroscopy will be discussed briefly.

In the next section an introduction to the ISS experiment and some theoretical background is presented. The femtosecond laser system and experimental details are discussed in section 3. In section 4, ISS results on intermolecular vibrational motion are presented and discussed. Time-resolved
ISRS observations of coherent molecular vibrations are presented in section 5 . In section 6 , the general occurrence of impulsive light-matter interactions is discussed and concluding remarks are made.

\section{The ISS experiment.}

2.1 DESCRIPTION AND THEORETICAL BACKGROUND. - The ISS experiment has been carried out in several configurations, but the most commonly used is the four-wave mixing geometry shown in figure 1. Two excitation pulses derived from the same laser, of central frequency and wave vectors $\left(\omega_{\mathrm{L}}, \mathbf{k}_{1}\right)$ and $\left(\omega_{\mathrm{L}}, \mathbf{k}_{2}\right)$, are overlapped spatially and temporally inside the sample to excite a coherent standing-wave response of frequency and wave vector $\left(\omega_{\alpha}, \mathbf{q}_{0}\right)$, where $\mathbf{q}_{0}=\mathbf{k}_{1}-\mathbf{k}_{2}$ and $\omega_{\alpha}(\mathbf{q})$ is the vibrational frequency of LS-active mode $\alpha$. The excitation mechanism is stimulated scattering, which occurs through mixing among the Fourier components contained within the necessarily broad spectral bandwidth of the ultrashort excitation pulses [8, 36]. The uncertainty-limited bandwidth must exceed the vibrational frequency for efficient ISS excitation to occur. This requirement can be recast in the time domain : the excitation laser pulse duration must be short compared to the vibrational oscillation period. The crossed excitation pulses then exert a spatially periodic, temporally impulsive driving force on the LS-active material mode. The (impulse) response to this force is a coherent, standing-wave oscillation.

\section{ISS PULSE SEQUENCE}

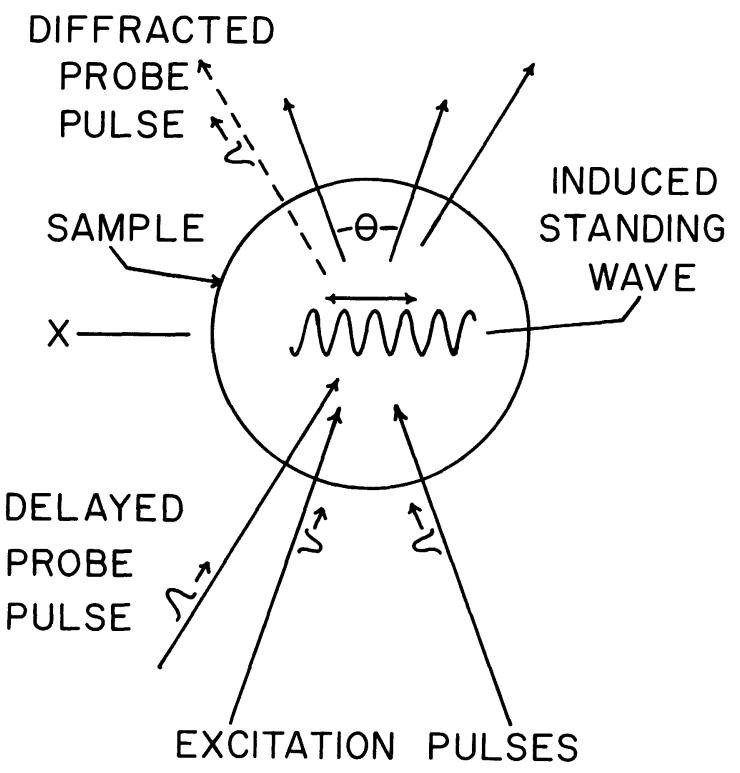

Fig. 1. - Schematic illustration of the impulsive stimulated scattering experiment. Two ultrashort, crossed excitation pulses "impulsively » excite a standing-wave material response which is monitored by coherent scattering («diffraction ») of the variably delayed probe pulse. 
The standing-wave vibrational displacements modulate the dielectric constant of the medium and can therefore be monitored by coherent scattering (i.e. « diffraction ») of variably delayed probe laser pulses which are incident at the phase-matching angle. See figure 1 . The time-dependent intensity of coherently scattered light undergoes damped oscillations from which vibrational frequency and dephasing information is deduced.

The main difference between ISS and time-resolved coherent anti-Stokes Raman scattering (CARS) or related techniques [37] is the arrangement through which excitation via stimulated scattering is carried out. In ISS, the frequency components of a single ultrashort laser output (which may or may not be split into two excitation pulses) are used. In CARS, two different, discrete laser frequencies are used. Material excitations are monitored by coherent scattering of variably delayed probe pulses in either case. In ISS, all the information including the fundamental vibrational frequency is extracted in the time domain. In CARS, the vibrational frequency is determined by the laser frequencies and vibrational dephasing information is extracted in the time domain. ISS provides a convenient means for femtosecond time-resolved study of low-frequency or heavily damped modes. CARS is better suited for study of high-frequency modes whose oscillation periods cannot be time-resolved [37].

A detailed theoretical description of ISS has been presented [8, 36]. The relevant results will be outlined here. The dielectric response, $\delta \varepsilon_{i j}(\mathbf{q}, t)$, to the electric field, $\mathbf{E}$, of the excitation pulses, is

$$
\begin{aligned}
\delta \varepsilon_{i j}(\mathbf{q}, t) & = \\
= & \int_{-\infty}^{\infty} \mathrm{d} t^{\prime} \sum_{k} G_{i j k l}^{\varepsilon \varepsilon}\left(\mathbf{q}, t-t^{\prime}\right) F_{k l}\left(\mathbf{q}, t^{\prime}\right),
\end{aligned}
$$

where $\mathbf{G}^{\varepsilon \varepsilon}$ is the impulse response function (Green's function) for the dielectric tensor and $F_{k l}(\mathbf{q}, t)$, the Fourier transform of

$$
F_{k l}(\mathbf{r}, t)=\frac{1}{8 \pi} E_{k}(\mathbf{r}, t) E_{l}(\mathbf{r}, t)
$$

can be thought of as the force exerted by the excitation pulses on the material dielectric. Note that ensemble averages of $\delta \varepsilon(q, t)$ and other material properties are implied in this treatment. $\mathbf{G}^{\varepsilon \varepsilon}$ is directly related to the dynamics of the LSactive material modes, i.e.,

$$
G_{i j k l}^{\varepsilon \varepsilon}(\mathbf{q}, t)=\sum_{\alpha} a_{i j}^{\alpha} a_{k l}^{\alpha} G^{\alpha}(\mathbf{q}, t),
$$

where $G^{\alpha}(\mathbf{q}, t)$ is the response function for (uncoupled) mode $\alpha$ and $a_{i j}^{\alpha}=\partial \varepsilon_{i j} / \partial Q^{\alpha}$ is the light-scattering coupling constant for mode $\alpha$ and light polarizations $i$ and $j$. The dielectric response in equation (1) is given directly by the vibrational displacements, $Q^{\alpha}$, i.e.

$$
\delta \varepsilon_{i j}(\mathbf{q}, t)=\sum_{\alpha} a_{i j}^{\alpha} Q^{\alpha}(\mathbf{q}, t)
$$

Equations (1) and (3) show that the force exerted by the excitation pulses on the material mode $\alpha$ is given by

$$
F^{\alpha}(\mathbf{q}, t)=\sum_{i j} a_{i j}^{\alpha} F_{i j}(\mathbf{q}, t)
$$

The material response to the excitation force is

$$
Q^{\alpha}(\mathbf{q}, t)=\int_{-\infty}^{\infty} \mathrm{d} t^{\prime} G^{\alpha}\left(\mathbf{q}, t-t^{\prime}\right) F^{\alpha}(\mathbf{q}, t)
$$

For collimated, ultrashort excitation pulses whose difference (scattering) wave vector is $\mathbf{q}_{0}$ and whose polarization directions are specified as $k$ and $l$, the excitation force can be written as

$$
F_{k l}(\mathbf{q}, t)=A_{k l} \delta(t)\left[\delta\left(\mathbf{q} \pm \mathbf{q}_{0}\right)+\delta_{k l} \delta(\mathbf{q})\right],(6)
$$

where $A_{k l}$ is a constant proportional to the excitation pulse intensity. In equation (6), the $\delta(\mathbf{q})$ term describes a spatially uniform force which produces a material response, as discussed below, that does not contribute to signal when the probe pulse is incident at the phase matching angle for «diffraction » from the standing-wave "grating " of wave vector $\pm q_{0}$. This term will be dropped below and reconsidered later. The propagation time of the excitation pulses through the sample is neglected in equation (6) but will be accounted for later as well.

The « impulsive » excitation force in equation (6) [with the $\delta(\mathbf{q})$ term omitted] yields a material response given directly by the impulse response function, i.e. from equations (1) and (6),

$$
\begin{aligned}
\delta \varepsilon_{i j}(\mathbf{q}, t) & =A_{k l} G_{i j k l}^{\varepsilon \varepsilon}(\mathbf{q}, t) \delta\left(\mathbf{q} \pm \mathbf{q}_{0}\right) \\
Q^{\alpha}(\mathbf{q}, t) & =a_{k l}^{\alpha} A_{k l} G^{\alpha}(\mathbf{q}, t) \delta\left(\mathbf{q} \pm \mathbf{q}_{0}\right)
\end{aligned}
$$

Thus, for a damped vibrational mode, $Q^{\alpha}(t) \propto$ $G^{\alpha}(t) \propto \mathrm{e}^{-\gamma_{\alpha} t} \sin \omega_{\alpha} t$, where $\gamma_{\alpha}$ is the damping rate and $\omega_{\alpha}$ is the frequency. Equation (8) shows that the material response to the crossed «impulsive » excitation pulses is a vibrational standing wave. If only one such mode is excited, then equation (3) shows that $\delta \varepsilon(\mathbf{q}, t) \propto Q^{\alpha}(\mathbf{q}, t)$.

For collimated, ultrashort incident and diffracted probe pulses whose polarization directions are specified as $j$ and $i$, respectively, ISRS signal intensity is given by

$$
I(\mathbf{q}, t) \propto\left(a_{i j}^{\alpha}\right)^{2}\left|\delta \varepsilon_{i j}(\mathbf{q}, t)\right|^{2} \propto\left|G_{i j k l}^{\varepsilon \varepsilon}(\mathbf{q}, t)\right|^{2} .
$$

If a single damped vibrational mode is excited and probed, then equation (9) shows that the ISS signal 
intensity oscillates at twice the vibrational frequency and decays at twice the vibrational dephasing rate, i.e. $I(t) \propto\left(\mathrm{e}^{-\gamma_{\alpha} t} \sin \omega_{\alpha} t\right)^{2}$.

Three polarization combinations were used in the experiments discussed below. "V-V » ISS experiments (analogous to polarized or $\mathrm{V}-\mathrm{V}$ spontaneous light scattering), in which the $G_{1111}^{\varepsilon \varepsilon}(t)$ component was measured, were carried out with vertically (V) polarized excitation and probe pulses and signal. « V-H » ISS experiments (analogous to depolarized light scattering), in which $G_{1212}^{\varepsilon \varepsilon}(t)$ was measured, were carried out with vertically and horizontally (V and $\mathrm{H})$ polarized excitation pulses, a $\mathrm{V}$-polarized probe pulse, and $\mathrm{H}$-polarized signal. « $\mathrm{V}-\mathrm{V} / \mathrm{H}-\mathrm{H}$ » ISS experiments (not directly analogous to any single light-scattering experiment), in which $G_{1122}^{\varepsilon \varepsilon}(t)$ was measured, were carried out with V-polarized excitation pulses and with the probe pulse and signal H-polarized.

To compare time-domain ISS data to frequencydomain, spontaneous LS spectra, we note that the latter can be described in the classical limit by [5]

$$
I(\mathbf{q}, \omega) \propto \frac{k_{\mathrm{B}} T}{\omega} \operatorname{Im}\left[G_{i j i j}^{\varepsilon \varepsilon}(\mathbf{q}, \omega)\right],
$$

where the incident and spontaneously scattered light polarization directions are specified as $i$ and $j$ and $\mathbf{G}^{\varepsilon \varepsilon}(\mathbf{q}, \omega)$, the Fourier transform of $\mathbf{G}^{\varepsilon \varepsilon}(\mathbf{q}, t)$, is related to material response functions $G^{\alpha}(\mathbf{q}, \omega)$ through the expression analogous to equation (2). Equations (9) and (10) show that, when the same components of the $\mathbf{G}^{\varepsilon \varepsilon}$ tensor are sampled in ISS and LS experiments, the information content of the data is identical in principle. However, depending on the nature of the modes under investigation, extraction of dynamical information may be more straightforward with one method than with the other. Detailed comparison of simulated ISS and LS data has been carried out $[8,36]$ and has indicated the utility of ISS for characterization of overdamped or weakly oscillatory modes such as intermolecular vibrations in liquids $[12,13]$, the topic discussed in section 4. Frequency-domain characterization of such modes is very difficult in spontaneous LS, although we note that stimulated Raman gain spectroscopy appears to offer considerably better prospects [24].
It is sometimes convenient to compare ISS and LS data through their connections to correlation functions rather than response functions. The LS spectrum is related to the dielectric constant time-correlation function, $\mathbf{C}_{i j k l}^{\varepsilon \varepsilon}(\mathbf{q}, t)=\left\langle\varepsilon^{i j^{*}}(\mathbf{q}, 0) \boldsymbol{\varepsilon}^{k l}(\mathbf{q}, t)\right\rangle$, in the classical limit through the fluctuation-dissipation expression

$\operatorname{Im}\left[\mathbf{G}^{\varepsilon \varepsilon}(\mathbf{q}, \omega)\right]=\frac{\omega}{2 k_{\mathrm{B}} T} \times \int_{-\infty}^{\infty} \mathrm{d} t \mathrm{e}^{j \omega t} \mathbf{C}^{\varepsilon \varepsilon}(\mathbf{q}, t)$.

ISS data yields $\mathbf{G}^{\varepsilon \varepsilon}(\mathbf{q}, t)$, from which $\mathbf{G}^{\varepsilon \varepsilon}(\mathbf{q}, \omega)$ and $\mathbf{C}^{\varepsilon \varepsilon}(\mathbf{q}, t)$ can be determined through Fourier transformations. A more direct connection to $\mathbf{C}^{\varepsilon \varepsilon}(\mathbf{q}, t)$ has been derived [36] :

$$
\mathbf{G}^{\varepsilon \varepsilon}(\mathbf{q}, t>0)=-\left(k_{\mathrm{B}} T\right)^{-1} \frac{\partial}{\partial t}\left[\mathbf{C}^{\varepsilon \varepsilon}(\mathbf{q}, t)\right],
$$

Thus the connection between ISS data and $C^{\varepsilon \varepsilon}(\mathbf{q}, t)$ is straightforward.

The microscopic contributions to $\mathbf{C}^{\varepsilon \varepsilon}(t)$ (or equivalently, $\left.\mathbf{G}^{\varepsilon \varepsilon}(t)\right)$ for Rayleigh and Raman spectra of molecular liquids have been discussed extensively $[5,6]$. The treatment is usually carried out in terms of a collective polarizability, $\boldsymbol{\Pi}$, and the associated time-correlation, $\mathbf{C}^{\Pi}(t)$, which is assumed to be proportional to $\mathbf{C}^{\varepsilon \varepsilon}(t)$. $\Pi$ is defined microscopically as a sum of single-molecule polarizabilities plus higher-order (« collision-induced») contributions. The connection between the macroscopic theory (in terms of $\varepsilon$ ) and the microscopic theory (in terms of I) is straightforward with the exception of local field corrections which do not concern us. Briefly, we can write $\mathbf{C}^{\Pi}(t)=\mathbf{C}_{0}(t)+\mathbf{C}_{2}(t)$, where $\mathbf{C}_{0}(t)$ and $\mathbf{C}_{2}(t)$ are the correlation functions for the isotropic and anisotropic parts, respectively, of the polarizability. Both $\mathbf{C}_{0}(t)$ and $\mathbf{C}_{2}(t)$ contribute to $\mathrm{V}-\mathrm{V}$ scattering, while V-H LS or ISS probes only $\mathbf{C}_{2}(t)$. The Rayleigh and Raman responses can be treated separately, i.e. $\mathbf{C}_{l}(t)=\mathbf{C}_{l}^{\text {Ray }}(t)+\mathbf{C}_{l}^{\text {Ram }}(t)$ where $l=0$ or 2 . Raman scattering is treated more easily since vibrational motions on different molecules are uncorrelated and so only single-molecule polarizabilities contribute. Assuming symmetric top molecules and neglecting vibration-rotation interactions,

$$
\mathbf{C}_{0}^{\mathrm{Ram}}(t) \propto \sum_{i=1}^{N}\left\langle Q_{i}^{\alpha}(t) Q_{i}^{\alpha}(0)\right\rangle=N\left\langle Q_{i}^{\alpha}(t) Q_{i}^{\alpha}(0)\right\rangle=N C_{i}^{\mathrm{vib}}(t)
$$

and

$$
\begin{aligned}
\mathbf{C}_{2}^{\operatorname{Ram}}(t) & \propto \sum_{i=1}^{N}\left\langle P_{2}\left[\hat{u}_{i}(t) \cdot \hat{u}_{i}(0)\right]\right\rangle\left\langle Q_{i}^{\alpha}(t) Q_{i}^{\alpha}(0)\right\rangle \\
& \propto N\left\langle\cos ^{2} \theta_{i}(t)-\frac{1}{3}\right\rangle\left\langle Q_{i}^{\alpha}(t) Q_{i}^{\alpha}(0)\right\rangle=N C_{i}^{\text {or }}(t) C_{i}^{\text {vib }}(t)
\end{aligned}
$$


for each Raman-active moleccular vibrational mode $\alpha$. The index $i$ labels the $i$-th molecule, $P_{2}$ is the second Legendre polynomial, $\hat{u}_{i}(t)$ is aligned with the molecular symmetry axis, and $\theta_{i}(t)$ is the angle between $\hat{u}_{i}(t)$ and $\hat{u}_{i}(0) . \quad C_{i}^{\text {vib }}(t)$, the singlemolecule vibrational correlation function, is given by the isotropic contribution to Raman scattering. The anisotropic contribution, "measured directly in depolarized Raman scattering, yields the product of $C_{i}^{\text {vib }}(t)$ and the single-molecule orientational correlation function, $C_{i}^{\text {or }}(t)$. Rayleigh scattering contains contributions from single-molecule polarizabilities, higher-order («collision-induced » or polarizabilities, and cross-terms (CR), i.e.

$$
\mathbf{C}_{2}^{\mathrm{Ray}}(t)=\mathbf{C}_{2}^{\mathrm{M}}(t)+\mathbf{C}_{2}^{\mathrm{CI}}(t)+\mathbf{C}_{2}^{\mathrm{CR}}(t)
$$

and

$$
\mathbf{C}_{0}^{\mathrm{Ray}}(t)=\mathbf{C}_{0}^{\mathrm{Cl}}(t)
$$

with $\mathbf{C}_{2}^{\mathrm{M}}(t)$ given by the orientational correlation function,

$$
\begin{aligned}
\mathbf{C}_{2}^{\mathrm{M}}(t) & \propto \sum_{i, j}^{N}\left\langle\left[P_{2}\left[\hat{u}_{i}(t) \cdot \hat{u}_{j}(0)\right]\right\rangle\right. \\
& =N(N-1)\left\langle P_{2}\left[\hat{u}_{i}(t) \cdot \hat{u}_{j}(0)\right]\right\rangle \\
& =N(N-1) C_{i j}^{\text {or }}(t) .
\end{aligned}
$$

$C_{i j}^{\text {or }}(t)$ includes single-molecule orientational correlations ( $i=j$ terms) as well as pair correlations $\left(i \neq j\right.$ terms). $\quad \mathbf{C}_{2}^{\mathrm{Cl}}(t)$ contains contributions from intermolecular translational and orientational motions. $\mathbf{C}_{2}^{\mathrm{CR}}(t)$ describes correlations between singlemolecule orientational alignment and intermolecular orientational and translational motions. These contributions to ISS signal will be discussed below.

\subsection{ISS WITH A SINGLE EXCITATION PULSE ; OPTICAL} KERR EFFECT CONFIGURATION. - In the case of a single, linearly polarized, collimated, ultrashort excitation pulse, the excitation force can be written from equation (6) as

$$
\begin{aligned}
F_{k l}\left(\mathbf{q}, t_{l}\right)=A_{k l} \delta_{k l} \delta\left(t_{l}\right) \delta & (\mathbf{q})= \\
& =A_{k k} \delta\left(t_{l}\right) \delta(\mathbf{q}),
\end{aligned}
$$

where the propagation time of the ( $k$-polarized) pulse through the sample has now been accounted for by defining a «local time » variable $t_{l}=t-$ $z n / c$, where $z$ is the direction of light propagation and $c / n$ is the speed of the light pulse inside the sample. The single pulse exerts a spatially uniform, temporally impulsive driving force on LS-active material modes. The response of the medium is given from equation (1) by

$$
\delta \varepsilon_{i j}\left(\mathbf{q}, t_{l}\right)=A_{k k} G_{i j k k}^{\varepsilon \varepsilon}\left(\mathbf{q}, t_{l}\right) \delta(\mathbf{q}) .
$$

For a single damped vibrational mode,

$$
\begin{aligned}
\delta \varepsilon_{i j}\left(\mathbf{q}, t_{l}\right) \sim G_{i j k k}^{\varepsilon \varepsilon}\left(\mathbf{q}, t_{l}\right) & \sim Q^{\alpha}\left(\mathbf{q}, t_{l}\right) \sim \\
& \sim \mathrm{e}^{-\gamma_{\alpha} t_{l}} \sin \omega_{\alpha} t_{l} \delta(\mathbf{q}) .
\end{aligned}
$$

Equation (19) describes a travelling-wave oscillation of frequency $\omega_{\alpha}$ and wave vector $\mathbf{q}_{\alpha}=\frac{\omega_{\alpha} n}{c} \hat{z}$. Thus a single ultrashort excitation pulse produces a coherent travelling-wave material reponse whose wave vector is parallel to that of the pulse. ISRS in this case can be understood as a forward-scattering process which is stimulated because the Stokesshifted light is contained within the frequency bandwidth of the pulse [35].

The coherent response, $\delta \varepsilon_{i j}\left(\mathbf{q}, t_{l}\right)$, can be probed in several ways with a linearly polarized probe pulse which is phase-matched for coherent scattering, i.e., which is collinear (or in practice, nearly collinear) with the excitation pulse and with the material excitation wave vector. The incident and coherently scattered probe light are collinear in this case, so measurement of the coherent scattering intensity as in figure 1 is impossible.

We discuss three different types of measurement thats can be carried out on the transmitted probe pulse - namely, measurement of its transit time through the sample, its polarization, or its spectral content - to monitor the coherent vibrational motion in the sample.

For a $k$-polarized probe pulse, the $\delta \varepsilon_{k k}\left(\mathbf{q}, t_{l}\right) \sim$ $G_{k k k k}^{\varepsilon \varepsilon}\left(\mathbf{q}, t_{l}\right)$ dielectric component is measured. The vibrational oscillations cause this component to oscillate (see Eq. (19)), and so the speed of light inside the medium oscillates. The amount of time required for the probe pulse to pass through the sample is an oscillatory function of the probe pulse delay relative to the excitation pulse, and can be determined by autocorrelation or other techniques [38].

For a $j$-polarized probe pulse, where $j \perp k$, the dielectric component $\delta \varepsilon_{j j}\left(\mathbf{q}, t_{l}\right) \sim G_{j j k k}^{\varepsilon \varepsilon}\left(\mathbf{q}, t_{l}\right)$ is monitored. In general its oscillations may have a different amplitude and sign than those of $\delta \varepsilon_{k k}\left(\mathbf{q}, t_{l}\right)$, but will cause a similar oscillatory behaviour in the probe pulse velocity. A probe pulse polarized at other angles, e.g. at $+45^{\circ}$ between $j$ and $k$, will experience an oscillatory birefringence due to the difference $\varepsilon_{j j}(t)-\varepsilon_{k k}(t)$. The transmission of the probe pulse through a polarizer oriented at $-45^{\circ}$ will oscillate at twice the vibrational frequency in such a case. For isotropic media, this (« optical Kerr effect ») experimental configuration probes $G_{j k j k}^{\varepsilon \varepsilon}\left(\mathbf{q}, t_{l}\right)$ and so yields the same result as the V-H ISRS configuration [39].

The probe pulse may also undergo oscillatory changes in its spectral content due to coherent scattering by the vibrational wave $[25,26,35]$. The 
unique effects that occur can be understood by consideration of the impulsive forces exerted by the excitation and probe pulses on the vibrational mode. Just like successive "pushes » of a pendulum, the two pulses exert driving forces on the vibrational mode which may be in or out of phase. If the probe pulse arrives in phase, i.e. an integral multiple of vibrational cycles after the excitation pulse, then it amplifies the vibrational motion, gives up energy to the vibrational wave, and emerges red-shifted. If the probe pulse arrives out of phase, it opposes the coherent vibrational motion, takes up energy from the vibrational wave, and emerges blue-shifted. The spectrum of the transmitted probe pulse therefore « wags » back and forth at the vibrational frequency. The theoretical treatment of this effect requires explicit consideration of the finite duration and corresponding spectral bandwidth of the probe pulse [35].

2.3 ISS WITH FINITE LASER PULSE DURATIONS. For the short-time responses of interest to us, ideal time resolution cannot always be assumed. The material response, $\delta \varepsilon_{i j}(t)$, is calculated from equation (1), which is a convolution of the impulse response function, $G^{\varepsilon \varepsilon}(t)$, with the excitation pulse temporal profile, $F_{k l}(t)$. The time-dependent signal, $I(t)$, is given to a good approximation by a convolution of $\left|\delta \varepsilon_{i j}(t)\right|^{2}$ with the probe pulse intensity profile, $P_{i j}(t)$, i.e.

$$
I(t)=\int_{-\infty}^{\infty} \mathrm{d} t^{\prime}\left|\delta \varepsilon_{i j}(t)\right|^{2} P_{i j}\left(t-t^{\prime}\right) .
$$

For damped vibrational modes, the effect of finite excitation pulse duration, $\tau_{\mathrm{L}}$, on the time-dependence of $\varepsilon_{i j}\left(t>\tau_{\mathrm{L}}\right)$ is merely to introduce a phase lag given by $\tau_{\mathrm{L}}$ and the vibrational dephasing rate [36]. In the fitting of data in section 4 , the phase lag (which never exceeded 15 fs [36]) was incorporated as an adjustable parameter in $\delta \varepsilon_{i j}(t)$, which was convoluted numerically over the probe pulse profile according to equation (20). For vibrational modes undergoing inhomogeneous dephasing, as discussed in section 4, finite excitation pulses can affect the apparent frequency and dephasing rate. For the data in section 4, these effects are negligible [36] so the procedure described above was used to generate all the fits in section 4.

\section{Experimental.}

A synchronously pumped and amplified femtosecond laser system, similar to one described earlier [40], was used. The system consists of two cw-pumped Nd : YAG lasers, a femtosecond dye laser, and a 3stage amplifier chain as illustrated in figure 2 . The first $\mathrm{Nd}$ : YAG laser is cw mode-locked $(41.1 \mathrm{MHz}$ Intra Action mode-locker) to produce $8 \mathrm{~W}$ of 1.06 -

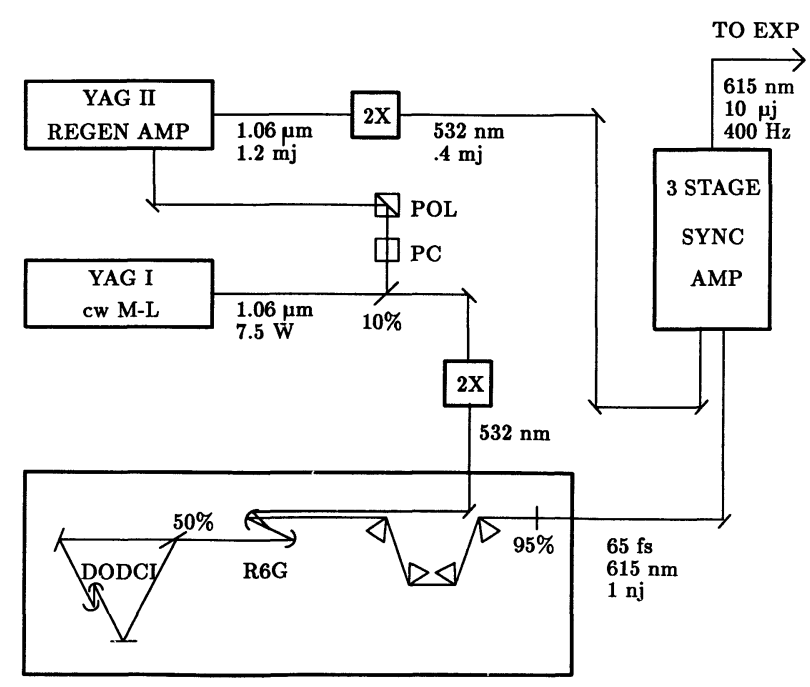

fs DYE LASER

Fig. 2. - Schematic diagram of the synchronously pumped and amplified femtosecond laser system. The first Nd : YAG laser (YAG I) is cw mode-locked and synchronously pumps the femtosecond dye laser. The second Nd : YAG laser (YAG II) regeneratively amplifies a single «seed" pulse from the YAG I output. The amplified, cavity-dumped output of YAG II is a single pulse which is frequency-doubled and used to synchronously amplify a femtosecond pulse from the dye laser. The amplified femtosecond pulse is used for ISS experiments. $P C \equiv$ Pockels' Cell ; Pol $\equiv$ Polarizer ; $2 \mathrm{X} \equiv$ Second harmonic generator ; $\mathrm{R} 6 \mathrm{G} \equiv$ Rhodamine $6 \mathrm{G}$ dye.

$\mu \mathrm{m}$ output consisting of 90 -ps pulses separated by about 12 ns. The output is frequency-doubled with a KTP doubling crystal (8 $\mathrm{mm}$ length, Aertron) to produce $1.9 \mathrm{~W}$ of $532-\mathrm{nm}$ light. About $1.2 \mathrm{~W}$ are used to synchronously pump the gain medium (a Rhodamine $6 \mathrm{G}$ dye jet) of the dye laser. The dye laser also includes a saturable absorber (DODCI dye jet) located in an anti-resonant ring. The 50-mW output of the dye laser consists of pulses of $65-\mathrm{fs}$ duration, separated by $\sim 12 \mathrm{~ns}$, whose central wavelength is $615 \mathrm{~nm}$ and whose spectral bandwidth is $\sim 9 \mathrm{~nm}$.

The Nd: YAG and dye laser cavity lengths must be matched precisely for synchronous pumping. To maintain optimal synchronization, the intensity of the dye laser is measured with a photodiode whose output is used to drive a feedback loop. This works because for a limited range of cavity lengths, the dye laser intensity decreases slightly when the dye laser cavity is slightly longer than the $\mathrm{Nd}$ : YAG cavity, and the dye laser intensity increases slightly when the dye laser cavity is too short. The most direct feedback arrangement would involve piezoelectric regulation of the dye laser cavity length [41]. We actually regulate the $41.1-\mathrm{MHz}$ frequency of the Nd : YAG mode-locker within a « window » of about $\pm 100 \mathrm{~Hz}$. The Nd: YAG output is unaffected by variation of the mode-locking frequency within this 
window, but the dye laser performance is very sensitive to adjustments of this magnitude. Stable operation can be maintained by this arrangement for very long times (sometimes hours), until finally the $\mathrm{Nd}$ : YAG « window » is exceeded by feedback-regulated frequency adjustments or by thermal drift in the Nd: YAG cavity length. This requires simple manual adjustment of a differential micrometer which adjusts the Nd: YAG cavity length.

A portion of the $1.06-\mu \mathrm{m}$ output of the first $\mathrm{Nd}$ : YAG laser is separated with a $10 \%$ reflector and a single pulse is selected at a $500-\mathrm{Hz}$ repetition rate by an electro-optic $\mathrm{Q}$-switch $\left(\mathrm{LiNiO}_{3}\right.$ crystal) with a homebuilt avalanche transistor driver. This pulse is used to «seed » a second Nd : YAG laser which acts as a regenerative amplifier. The seeding is accomplished acousto-optically [42] rather than electro-optically as has been reported earlier [40]. An acousto-optic Q-switch inside the second laser cavity is used to prevent lasing until it is switched off at the same time as the «seed " pulse from the first laser is selected. The seed pulse is aligned to coincide in space with the Bragg-diffracted output of the Qswitch, and so the seed is diffracted into the second Nd : YAG laser cavity by the Q-switch before the Qswitch is completely «off ». The 90-ps seed pulse is then amplified by about 50 round trips through the second Nd: YAG cavity. Finally, the pulse is cavitydumped by an electro-optic Q-switch $\left(\mathrm{LiNiO}_{3}\right.$, In$\mathrm{rad}$ ) inside the second Nd : YAG cavity. The output is a $1.06-\mu \mathrm{m}, 1.1-\mathrm{mJ}, 90-\mathrm{ps}$ pulse with a $500-\mathrm{Hz}$ repetition rate. This pulse is synchronized precisely with a dye laser pulse and therefore can be used for synchronous amplification.

The regeneratively amplified output is frequencydoubled in a KTP doubling crystal $(5 \mathrm{~mm}$ length, Aertron) to yield $450 \mu \mathrm{J}$ of 532 -nm light. This is split into three portions by $4 \%$ and $20 \%$ partial reflectors, and the three portions are used in order of increasing energy to longitudinally pump three amplifier stages. Each stage consists of a $1-\mathrm{cm}$ flowing dye cell with sulforhodamine 640 dye. Saturable absorber (malachite green dye) jets separate the successive amplification stages. The output is compressed with a grating pair ( $\sim 60 \%$ throughput $)$ to yield $60-\mathrm{fs}, 615-\mathrm{nm}, 6-\mu \mathrm{J}$ pulses at a $500-\mathrm{Hz}$ repetition rate with about $\pm 5 \%$ peak-to-peak energy fluctuations.

For most of the experiments reported here, the amplified output was filtered extensively $(\sim 10 \times)$ to avoid any intensity-dependent effects on signal. The output was split three ways to yield the excitation and probe pulses. These were polarized $\mathrm{V}$ or $\mathrm{H}$ relative to the scattering plane formed by the excitation pulses, focussed to $200-\mu \mathrm{m}$ spot sizes, and crossed in a noncoplanar geometry with a $5^{\circ}$ angle between excitation pulses inside a $2-\mathrm{mm}$ spectrophotometer cuvette filled with the liquid sample.
In some experiments the sample temperature was regulated in a closed-cycle refrigerative cooler with a P-I-D controller (Lakeshore). The variable delay of the probe pulse was controlled by a $1-\mu \mathrm{m}$ steppingmotor delay line. The energy of each pulse was measured, and pulses whose energies fell outside an energy range of about $\pm 1 \%$ were rejected. The signal from the remaining pulses was stored and averaged. Typically, about 200 repetitions were averaged at each point on the delay line.

\section{Intermolecular vibrational motion in simple molecular liquids.}

In this section results are presented which demonstrate unambiguously the vibrational character of local intermolecular motions in liquids. Almost all the data shown are from liquid carbon disulphide $\left(\mathrm{CS}_{2}\right)$, although similar data have been recorded from many molecular liquids by us [11-14] and also by Kenney-Wallace et al. [15, 16]. The results of ISS experiments on pure $\mathrm{CS}_{2}$ liquid at room temperature with several experimental configurations are shown first. Data from $\mathrm{CS}_{2}$ at various temperatures between $165 \mathrm{~K}$ and $300 \mathrm{~K}$ are then presented. These results are most interesting because at low temperatures weakly oscillatory time-dependent responses are observed, leaving no doubt about the basic interpretation of the data in terms of intermolecular vibrational motion. These results also suggest the importance of inhomogeneous vibrational dephasing and permit estimation of the extent of inhomogeneity in the intermolecular vibrational frequency. Data are then shown from $\mathrm{CS}_{2} /$ cyclohexane solutions at room temperature. These data indicate, along with other considerations, that intermolecular librational (as opposed to translational) motions dominate the ISS response.

ISS data from pure benzene liquid at room temperature are also shown to illustrate the general nature of the effects under observation.

4.1 PuRE $\mathrm{CS}_{2}$ LIQUid - ROOM TEMPERATURE. Figure 3 shows V-H ISS data recorded from pure $\mathrm{CS}_{2}$ liquid at room temperature. The signal shows an initial feature centred at $t=0$ which is due to the essentially instantaneous electronic response to the nonresonant pulses. This feature, which is shown by all materials, persists only while all three pulses are inside the sample at the same time. Its initial temporal profile nearly matches that obtained from pure $\mathrm{H}_{2} \mathrm{O}$ liquid (broken curve), which shows only an electronic response. However, in $\mathrm{CS}_{2}$ the signal continues to increase after the $t \approx 0$ electronic signal is well past its maximum. This reflects the inertial, noninstantaneous response of the $\mathrm{CS}_{2}$ molecules to the excitation pulses. The pulses exert sudden (nearly "impulsive") torques on molecules, inducing molecular orientational motion and net molecu- 


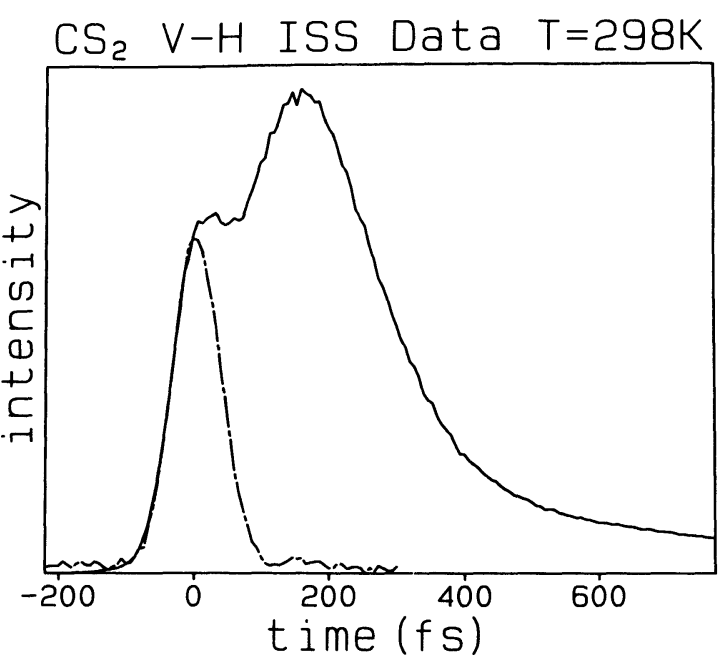

Fig. 3. - V-H ISS data from $\mathrm{CS}_{2}$ liquid. The signal after $t=0$ continues to rise, indicating the inertial character of short-time motion in the fluid. After reaching a maximum, the signal decreases rapidly and then more slowly. The broken curve is V-H ISS data from $\mathrm{H}_{2} \mathrm{O}$, recorded immediately after the $\mathrm{CS}_{2}$ data, under identical experimental conditions.

lar alignment, through the single-molecule polarizabilities (i.e., through stimulated rotational Raman scattering). The pulses also exert forces on molecule pairs, triplets, etc., inducing intermolecular translational and librational motions, through higherorder polarizabilities [1-6] (i.e., through stimulated «collision-induced» scattering). The orientational and intermolecular responses cannot occur instantaneously, however. The gradual increase in ISS signal after the $t \approx 0$ electronic response is ending (between $t \approx 70 \mathrm{fs}$ and $t \approx 170 \mathrm{fs}$ in the data) is a direct manifestation of inertial molecular motion away from equilibrium which continues after the excitation pulses have left the sample. After reaching a maximum, the signal decreases rapidly and then more slowly. The rapid decay is due, we believe, to a reversal in the directions of molecular motions which results primarily from intermolecular repulsions between "colliding " molecules. In other words, the short-time data reflect intermolecular vibrational motion in the liquid. This motion was not apparent in earlier subpicosecond time-resolved data from $\mathrm{CS}_{2}$ and other liquids [43-46], which revealed two distinct decay rates but not the shorter-time inertial rise in signal. Spontaneous LS spectra of $\mathrm{CS}_{2}$ show some structure in the Rayleigh wing which suggests inertial motion $[18,20,30,47,48]$ but which is difficult to analyse quantitatively. This structure was revealed far more clearly in stimulated Raman gain spectra [24], which were interpreted tentatively (and we believe correctly) in terms of intermolecular librations.

We have attempted to interpret the data in terms of simple physical models with a small number of adjustable parameters. We envision the molecules as occupying local potential minima in the "cavities » formed by their immediate neighbours. Motion about these minima should have vibrational character $[1-6,18-24,32-34,49-58]$. Vibrational phase coherence can be lost rapidly due to phase-changing and dissipative collisions (i.e. homogeneous dephasing) and due to differences in local intermolecular vibrational frequencies (i.e. inhomogeneous dephasing). The relative importance of these two dephasing contributions has been discussed at some length [34, 49-51]. We originally fit ISS data from $\mathrm{CS}_{2}$ at room temperature to a homogeneous dephasing model $[11,12]$, using an overdamped oscillator functional form to describe the response at short times and a relaxational reponse to describe the long-time «collective» orientational motion through which the fluid returns to the isotropic state. The functional form of the response function (before convolution as described in Sect. 2.3) used to generate the fit was

$$
G^{\varepsilon \varepsilon}(t)=A\left(\mathrm{e}^{-\gamma_{1} t}-\mathrm{e}^{-\gamma_{2} t}\right)+B \mathrm{e}^{-\Gamma t},
$$

where $\gamma_{2,1}=\gamma \pm\left(\gamma^{2}-\omega_{0}^{2}\right)^{1 / 2}$ and $\omega_{0}$ is the (undamped) natural vibrational frequency, $\Gamma$ is the collective orientational relaxation rate (corresponding to the width of the narrow, approximately Lorentzian part of the Rayleigh line) and $A$ and $B$ are amplitudes which depend on single-molecule and higher-order polarizabilities. It was later shown [13] that good fits to the data could also be obtained with an underdamped oscillator functional form,

$$
G^{\varepsilon \varepsilon}(t)=A \mathrm{e}^{-\gamma t} \sin \omega t+B \mathrm{e}^{-\Gamma t},
$$

where $\omega=\left(\omega_{0}^{2}-\gamma^{2}\right)^{1 / 2}$. Finally, quite good fits are also obtained (as shown below) with a functional form based (with approximations that have been discussed) on a model of purely inhomogeneous dephasing $[13,14]$ :

$$
G^{\varepsilon \varepsilon}(t)=A \mathrm{e}^{-\Delta^{2} t / 2} \sin \omega_{A} t+B \mathrm{e}^{-\Gamma t},
$$

where $\Delta$ is the spread (approximate root mean square deviation) of vibrational frequencies about the average undamped frequency, $\omega_{A}$, assuming a nearly Gaussian frequency distribution [13, 14]. The conclusion drawn from the room-temperature data is that the intermolecular vibrational character of the short-time motion is clearly indicated, but a quantitative description of the response cannot be given uniquely.

Similar time-dependent responses are observed in $\mathrm{V}-\mathrm{V}, \mathrm{V}-\mathrm{V} / \mathrm{H}-\mathrm{H}$, and OKE experimental configurations, as shown in figures 4-6. These data do not permit more critical evaluation of the response functions in equations (21)-(23). As an interesting aside, we draw attention to the previously unpub- 


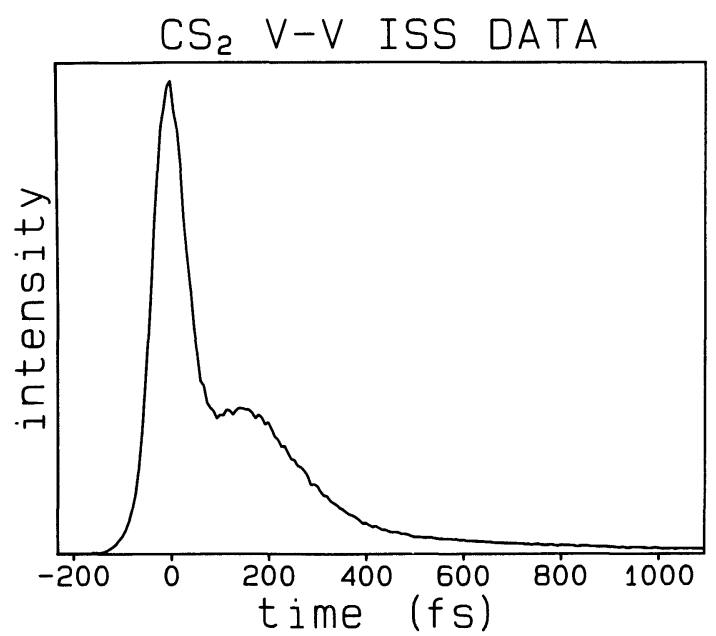

Fig. 4. - V-V ISS data from $\mathrm{CS}_{2}$ liquid. The data are similar in form to that in figure 3 . The relative contribution of the purely electronic response is larger in this configuration.

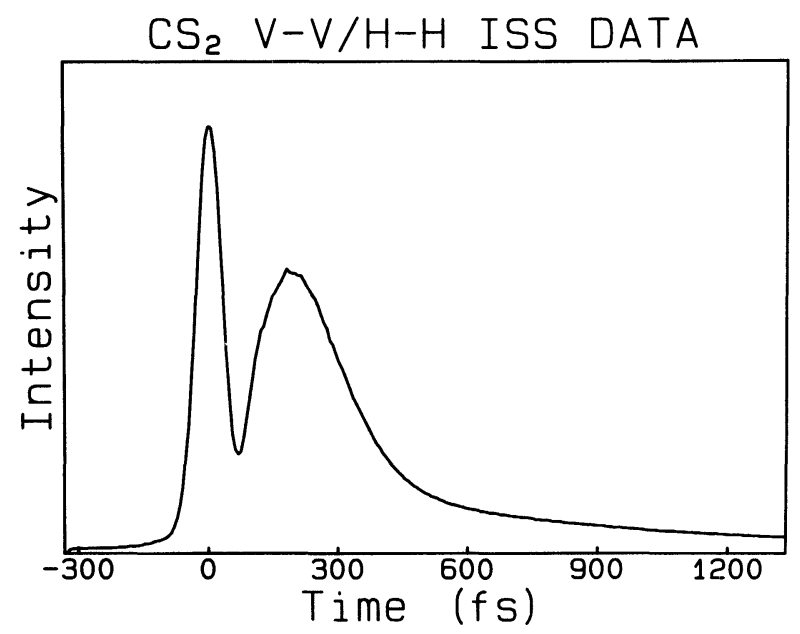

Fig. 5. - V-V/H-H ISS data from $\mathrm{CS}_{2}$ liquid. The electronic and nuclear responses are similar to those observed in figures 3 and 4 , but they lead to dielectric responses of opposite sign. Their cancellation at $t \geq 0$ results in the sharp « dip » in signal intensity.

lished $\mathrm{V}-\mathrm{V} / \mathrm{H}-\mathrm{H}$ data shown in figure 5. The sharp « dip » in signal intensity shortly after $t=0$ is due to cancellation of the electronic and nuclear responses in the $\mathrm{V}-\mathrm{V} / \mathrm{H}-\mathrm{H}$ configuration. The origin of this effect is well known [39, 59-61]. The V-polarized excitation pulses produce an electronic polarization in the $\mathrm{V}$ direction which results in an increase in both the parallel $\left(\varepsilon_{11}\right)$ and perpendicular $\left(\varepsilon_{22}\right)$ dielectric components, i.e., $\delta \varepsilon_{11}^{\text {elec }}=3 \delta \varepsilon_{22}^{\text {elec }}>0$. The excitation pulses also produce a (delayed) molecular orientational alignment, in the V-direction. The resulting change in $\varepsilon_{11}$ due to molecular orientation, $\delta \varepsilon_{11}^{\text {or }}$, is positive, but $\delta \varepsilon_{22}^{\text {or }}$ is negative, i.e. $\delta \varepsilon_{11}^{\text {or }}=$ $-2 \delta \varepsilon_{22}^{\text {or }}$ and $\delta \varepsilon_{11}^{\text {or }}>0$. Thus in the V-V ISS data

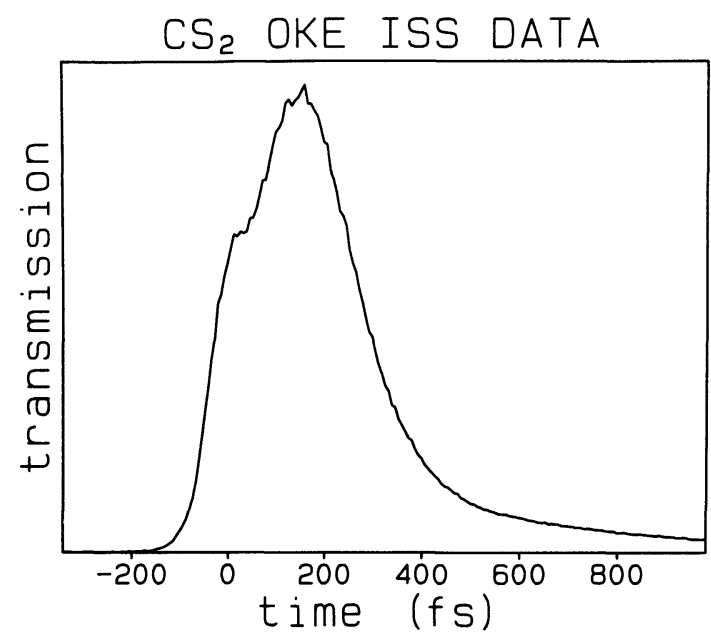

Fig. 6. - Optical Kerr effect (OKE) data from $\mathrm{CS}_{2}$ liquid. The data are similar in form to those in figures 3 and 4 .

shown in figure 4 , the initial change $\delta \varepsilon_{11}^{\text {elec }}(t)$ is of the same sign as $\delta \varepsilon_{11}^{\text {or }}(t)$, and the signal, which depends on $\left[\delta \varepsilon_{11}(t)\right]^{2}$, is never diminished due to a cancellation between the two contributions. On the other hand, the V-V/H-H data show a dramatic « dip » as the excitation pulses are leaving the sample, because the magnitude of $\delta \varepsilon_{22}^{\text {elcc }}(t)$ is decreasing toward zero while the magnitude of $\delta \varepsilon_{22}^{\text {or }}(t)$ is increasing.

\subsection{Pure $\mathrm{CS}_{2}$ - Temperature DePendence. -} Figure 7 shows V-H ISS data recorded from $\mathrm{CS}_{2}$ liquid at various temperatures between $165 \mathrm{~K}$ and $300 \mathrm{~K}[10,11]$. The data show that as the $\mathrm{CS}_{2}$ temperature is lowered, the time-dependent response of the liquid becomes weakly oscillatory. To illustrate this more clearly, the insets to figure 7 show the temporal region $t>400 \mathrm{fs}$ of each sweep on an expanded vertical scale. Figure 8 shows semilog plots of each sweep. The weakly oscillatory character of the data at $T \leqslant 240 \mathrm{~K}$ is unmistakable and reproducible. The time-dependence of signal is independent of laser intensity at the intensity levels used. Similar data, also showing the weak oscillation at $T \leqslant 240 \mathrm{~K}$, were recorded in $\mathrm{V}-\mathrm{V}$ and OKE ISS experiments. These data demonstrate unambiguously that the physical origin for the short-time response is intermolecular vibrational motion, as concluded originally $[11,12]$.

All of the data in figures 7 and 8 are fit based on the inhomogeneous dephasing response function in equation (23) (dashed curves). It is clear that the fits are excellent, within signal/noise at almost all times. The broken curves in figure 7 show fits based on the damped-oscillator response function in equation (22). Although all of the qualitative features in the data can be reproduced by equation (22), the weak oscillation cannot be fit accurately at any temperature $T \leqslant 240 \mathrm{~K}$. The additional temporal structure in 


\section{$\mathrm{CS}_{2}$ T-Dependent ISS Data}

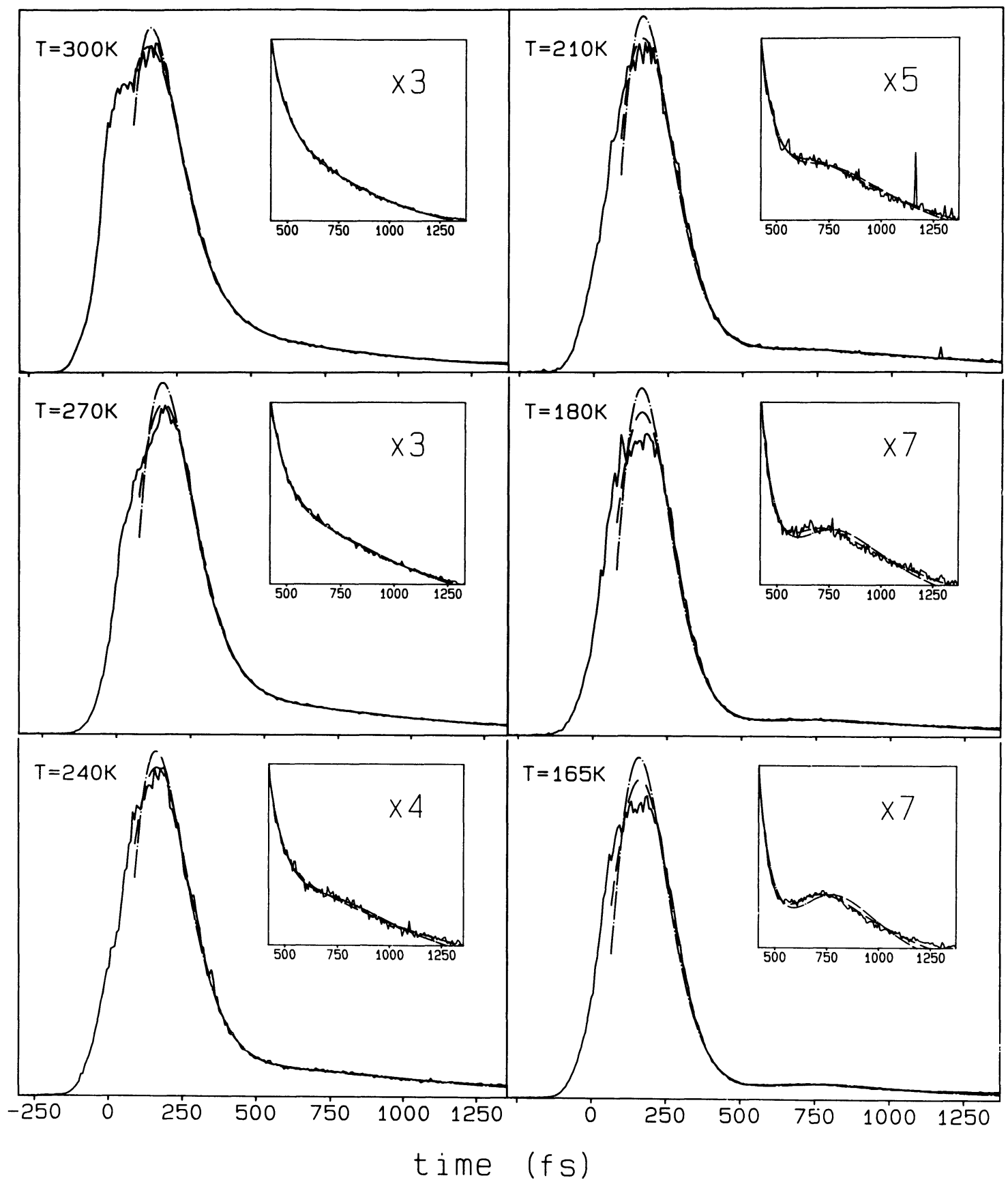

Fig. 7. - V-H ISS data (solid curves) from $\mathrm{CS}_{2}$ liquid at various temperatures. Each scan shows the inertial rise in signal after $t=0$ as in figures 3-6. At lower temperatures, the rapid decay is followed by a weak oscillatory feature attributable to oscillatory intermolecular librational motion in the liquid. The temporal region at which this occurs is shown in the Inset to each scan. The dashed curves show fits based on a model of entirely inhomogeneous dephasing, generated using equation (23), convoluted as described in the text. Fits based on equation (22) (broken curves) illustrate the inadequacy of a model of entirely homogeneous dephasing. Note that the time scale under each scan indicates $t=0$ as the time to which the dashed-curve fit extrapolates.

the low-temperature data permits far more critical analysis of the different response functions. The temperature-dependent data strongly suggest that inhomogeneous dephasing is the main cause of rapid loss of vibrational coherence. It is expected to be large, reflecting the wide range of local molecular configurations in the liquid and the range of different intermolecular motions that are possible even within a single configuration. Of course homogeneous dephasing due to intermolecular collisions, which should also be rapid, may still contribute significantly to the responses we observe. We have neglected it 


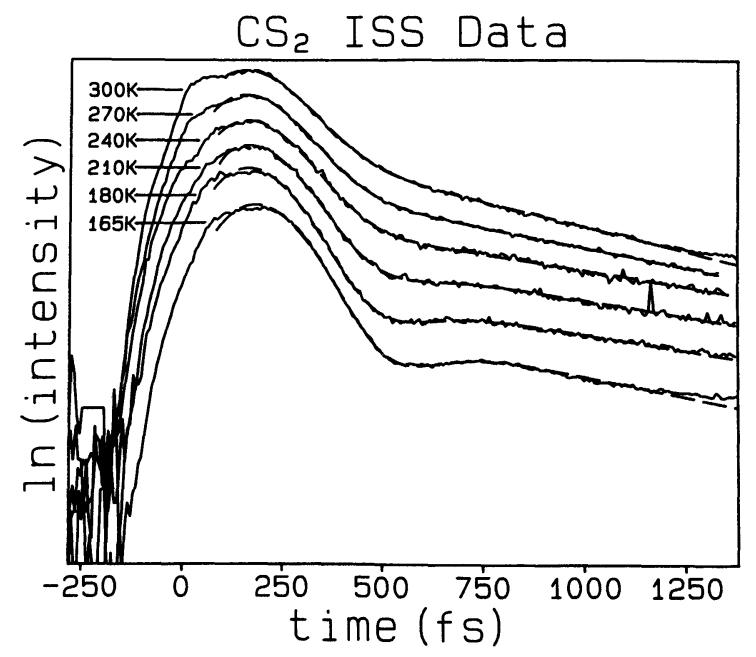

Fig. 8. - Temperature-dependent V-H ISS data (solid curves) from $\mathrm{CS}_{2}$ liquid, plotted on a logarithmic vertical scale. The oscillatory feature is evident at the lowest four temperatures. The data and fits (dashed curves) are the same as those in figure 7.

here to minimize the complexity (and number of adjustable parameters) of our model, and because the data can be fit well without it.

The temperature-dependent parameters determined by the fits are listed in table I. The average intermolecular vibrational frequency, $\omega_{A}$, and the range in frequencies, $\Delta$, are the quantities of primary interest here. We believe that within the context of this simple model, the values of $\omega_{A}$ and $\Delta$ at $T<240 \mathrm{~K}$ are accurate to within $\pm 10 \%$. At $T=240 \mathrm{~K}$, the oscillatory feature is subtle and the parameters are probably good to only $\pm 30 \%$. At $T \geqslant 270 \mathrm{~K}$, the uncertainties are about $\pm 50 \%$. Table I shows that $\omega_{A}$ increases and $\Delta$ decreases as the temperature is reduced. We believe that the former trend may be attributable mainly to the substantial $(13 \%)$ increase in density of the liquid as the temperature is lowered $[47,50,51,62]$. Since the frequency is likely determined largely by intermolecular repulsions, reduction in the size of the «cavity " around a molecule should lead to larger frequencies. The decrease in inhomogeneity is plaus-

Table I. - Fitting parameters for temperature-dependent ISS data from $\mathrm{CS}_{2}$ liquid.

\begin{tabular}{cccccc}
\hline$T(\mathrm{~K})$ & $\omega_{A}\left(\mathrm{ps}^{-1}\right)$ & $\Delta\left(\mathrm{ps}^{-1}\right)$ & $\Gamma\left(\mathrm{ps}^{-1}\right)$ & $A$ & $B$ \\
\hline 300 & 3.4 & 5.7 & 0.94 & 16 & 5.6 \\
270 & 3.9 & 5.7 & 0.76 & 15 & 4.8 \\
240 & 5.3 & 5.2 & 0.62 & 12 & 4.3 \\
210 & 6.6 & 4.5 & 0.61 & 10 & 4.1 \\
180 & 7.0 & 4.3 & 0.60 & 10 & 3.8 \\
165 & 7.2 & 4.0 & 0.77 & 9 & 4.1 \\
\hline
\end{tabular}

ible since at lower temperatures the liquid has access to fewer local configurations.

At each temperature, additional scans were run out to $6 \mathrm{ps}$ delay. The data at long times $(>1 \mathrm{ps})$ will be discussed in more detail subsequently since our emphasis at present is on the short-time behaviour. We mention here three temperature-dependent trends observed in the «long-time" ( $>1 \mathrm{ps}$ ) response. First, its relative intensity in the data decreases as $T$ decreases, presumably because the liquid at low $T$ is more viscous and therefore less compliant to the local motions induced by the excitation pulses. Second, the signal persists for longer times at lower $T$, i.e. the fluid requires longer times to return to the isotropic state. Third, the longtime response decays more or less exponentially at room $T$, but deviates from single-exponential decay as $T$ is reduced. This is in accord with the nonLorentzian central Rayleigh line observed in $\mathrm{CS}_{2}$ at low $T[30,48]$. The «long-time » response, i.e. the part of the signal not described by the first term in equation (23), can be fit approximately with two relaxation times, as has been observed in other liquids by Lotshaw et al. [16]. Our data do not allow us to conclude that two relaxation times are sufficient ; a continuous distribution of relaxation times may be more appropriate [7]. For present purposes, the values of $\Gamma$ in table I describe only the first part of the long-time response, and cannot be interpreted as collective reorientation times.

We wish to discuss the type of intermolecular motion under observation. For several reasons, we believe the response observed to be dominated by rotational molecular motions (which show vibrational, i.e. librational, character) rather than translational molecular motions. Contributions to scattering which arise from only single-molecule polarizabilities (i.e. orientational motions induced by the excitation pulses which lead to net molecular alignment detected by the probe pulse ; see equation (16)) are due exclusively to orientational motions. Contributions to signal which arise from «crossterms " between single-molecule and higher-order polarizabilities (i.e. orientational motions induced by the excitation pulses which lead to librational « collisions » detected by the probe pulse, or intermolecular motions induced by the excitation pulses which lead to net molecular alignment detected by the probe pulse) are due predominantly to rotation. Only a part of the remaining signal arising from only higher-order polarizabilities is due to translational intermolecular motions [1-6, 30, 63-66]. Recent molecular dynamics simulations indicate that in $\mathrm{CS}_{2}$, the V-H LS (and therefore ISS) signal is due mainly to single-molecule polarizabilities [67]. The similarity between the spontaneous depolarized Rayleigh and Raman wings in $\mathrm{CS}_{2}$ (and other molecular liquids) [20, 28-30, 47], and the similarity 
between $\mathrm{V}-\mathrm{V}$ and $\mathrm{V}-\mathrm{H}$ data, offer some further evidence of interest. Finally, in other ISS experiments carried out on $\mathrm{CS}_{2}$ /hydrocarbon solutions, discussed below, very little change is observed in the short-time dynamics as the $\mathrm{CS}_{2}$ is diluted to as little as $1 \%$. At low concentration, all the signal arises from $\mathrm{CS}_{2}$ single-molecule polarizabilities which probe only rotational motion.

Assuming that rotational motion is being observed, then the short-time vibrational response reflects intermolecular librational motions, somewhat similar to the motions involved in librational optic phonons in molecular crystals. Of course in the liquid there are many such motions rather than a few « normal» modes as in a crystal. Nevertheless, a physically meaningful configuration-averaged intermolecular librational frequency is determined from our measurements of $\omega_{A}$. The values of $\Delta$ also have a simple physical meaning, i.e. they indicate the extent of inhomogeneity in the intermolecular librational frequency. An average librational force constant, whose magnitude measures the restoring torque against rotational motion felt by a molecule due to interactions with its neighbours, can be determined from $\omega_{A}$, and its inhomogeneity from $\Delta$ $[13,14]$. Thus the intermolecular vibrational spectroscopy which has been carried out in the time domain yields basic information about both molecular dynamics and intermolecular forces.

4.3 $\mathrm{CS}_{2}$ /CYClOHEXANE SOLUTIONS. - Figure 9 shows previously unpublished $\mathrm{V}-\mathrm{H}$ ISS data from solutions of $\mathrm{CS}_{2}$ in cyclohexane, with $\mathrm{CS}_{2}$ volume fractions ranging from $1 \%$ to $80 \%$. A more detailed

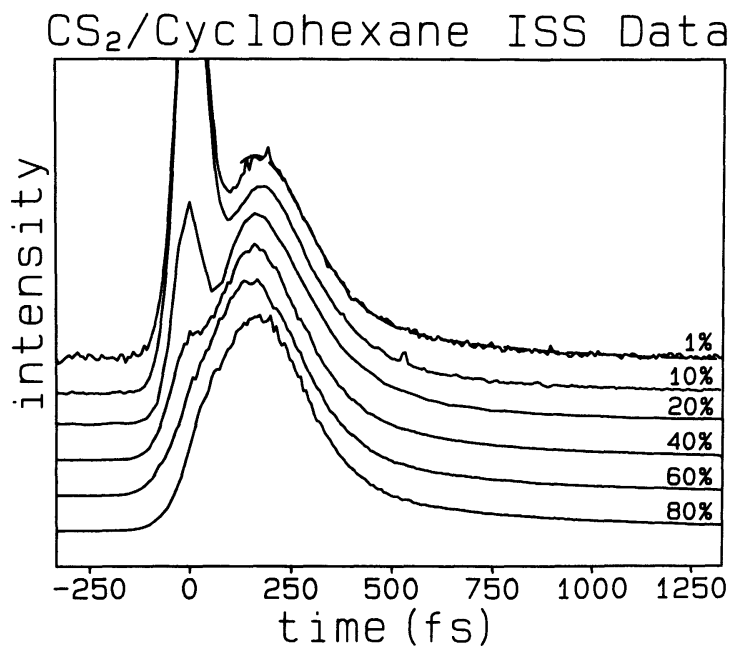

Fig. 9. - V-H ISS data from $\mathrm{CS}_{2}$ /hydrocarbon solutions of various volume fractions. The short-time response shows very little concentration dependence. The fit to the $1 \%$ data is based on equation (23), with parameters $\omega_{A}=2.3 \mathrm{ps}^{-1}, \Delta=3.8 \mathrm{ps}^{-1}, \Gamma=1.1 \mathrm{ps}^{-1}, A / B=2.8$. Fits to the other data were almost indistinguishable and yielded parameters that were identical within uncertainty. analysis of this data will be presented subsequently. For present purposes, we wish to emphasize the similarity in the short-time responses among all of the data. The data do not indicate significant concentration-dependent changes in the intermolecular vibrational dynamics. As discussed above, the lowconcentration short-time response must arise exclusively from librational motions of the $\mathrm{CS}_{2}$ molecules against their cyclohexane neighbours. The similarity of the time-dependent responses at all concentrations suggests, in addition to other evidence discussed above, that librational motions give the predominant contribution to signal even in pure $\mathrm{CS}_{2}$.

4.4 BenZENE. - We show in figure $10 \mathrm{~V}$-H ISS data collected from benzene liquid at room temperature [13]. The inset shows the same data plotted with a logarithmic vertical scale. As in the low-temperature $\mathrm{CS}_{2}$ data, the data clearly show weakly oscillatory behaviour due to intermolecular vibrational motion. Raman spectra suggest similar dynamics [28], indicating that librational motion dominates the ISS response in benzene as well as in $\mathrm{CS}_{2}$. This data provides an additional example of the shorttime behaviour under consideration. We note that data similar to that obtained from room-temperature $\mathrm{CS}_{2}$ liquid, which is nonoscillatory but clearly shows inertial short-time motion, has been recorded from several other liquids by us [13] and by Lotshaw et al. [16]. We expect these short-time responses to appear in most molecular liquids.

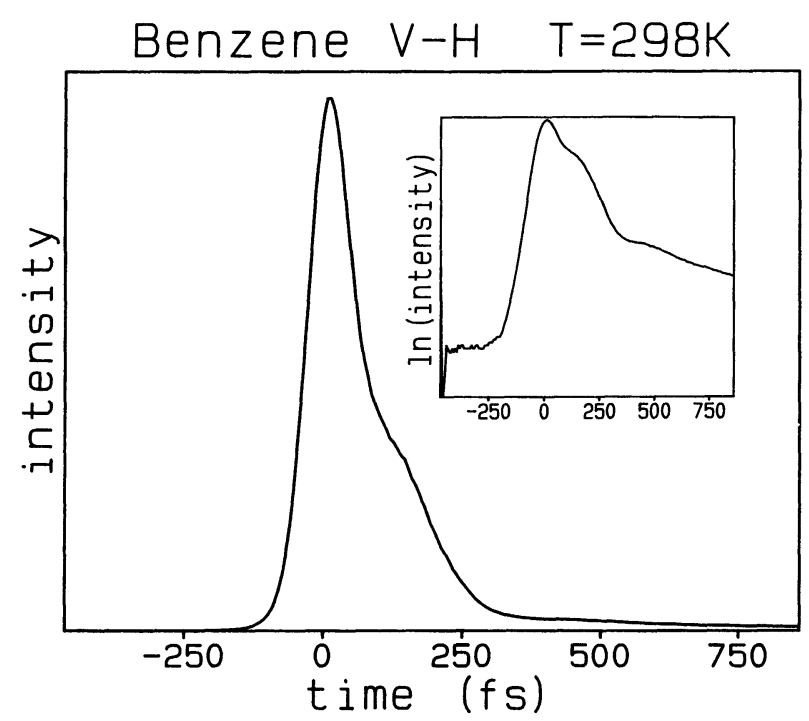

Fig. 10. - V-V data from benzene liquid at room temperature. The Inset shows a logarithmic plot of the data. The response is weakly oscillatory, as in $\mathrm{CS}_{2}$ at low temperatures.

4.5 SUMMARY. - The observations of weakly oscillatory time-dependent ISS responses in $\mathrm{CS}_{2}$ liquid at low temperatures and benzene liquid at room tem- 
perature confirm unambiguously the interpretation of short-time ISS data in molecular liquids in terms of intermolecular vibrational motions. It is clear that many liquids will display this type of behaviour. The low-temperature responses in $\mathrm{CS}_{2}$ suggest a major role played by inhomogeneous intermolecular vibrational dephasing. Our $T$-dependent values of $\omega_{A}$ and $\Delta$ can be interpreted physically in terms of the configuration-averaged librational frequency and the extent of inhomogeneity in that frequency.

\section{Coherent molecular vibrational motion.}

Here we present data illustrating the extension of impulsive stimulated Raman scattering (ISRS) to time-resolved observation of coherent intramolecular vibrational motion [25-26]. ISRS data from several organic molecules in the liquid phase, collected using a variety of experimental configurations, are presented. The ISRS technique permits direct observation of molecular vibrational motion and, in some cases, molecular orientational motions as well. ISRS also opens up prospects for time-resolved spectroscopic measurements to be carried out on vibrationally distorted (i.e., stretched or bent) molecules whose nonequilibrium configurations at the time of observation are narrowly distributed and can be known with reasonable accuracy.

To illustrate some of the experimental configurations discussed earlier for time-resolved observation of molecular vibrational motion, we present ISRS data collected from dibromomethane liquid using four different arrangements. Analysis of frequencydomain Rayleigh and Raman spectra of dibromomethane has indicated that the molecular motion can be described approximately in terms of that of a symmetric top [68], as discussed in section 2.1. Figures 11 and 12 show V-V and V-H ISRS data, respectively, collected with the experimental configuration shown in figure 1. Both scans show pronounced temporal oscillations in signal intensity. The oscillation period is $190 \mathrm{fs}$, which matches the known 5.2-THz frequency of the $\left(173-\mathrm{cm}^{-1}\right) \mathrm{Br}-\mathrm{C}-$ $\mathrm{Br}$ halogen bending mode of $\mathrm{CH}_{2} \mathrm{Br}_{2}$ [69]. Thus coherent vibrational motion is induced through ISRS excitation and monitored in the time domain.

The data in figures 11 and 12 show additional features as well as the high-frequency oscillations. As in data shown earlier, the initial sharp rise in signal at $t \approx 0$ is due to the essentially instantaneous electronic response of the molecules to the nonresonant excitation pulses. More important to us are significant contributions to signal at $t>0$ due to intermolecular motions and molecular orientational motions induced by the excitation pulses, i.e. the Rayleigh response. In other words, the same effects that occur in $\mathrm{CS}_{2}$ and benzene also occur in dibromomethane, in addition to the high-frequency intramolecular vibrations. Since we are not analysing

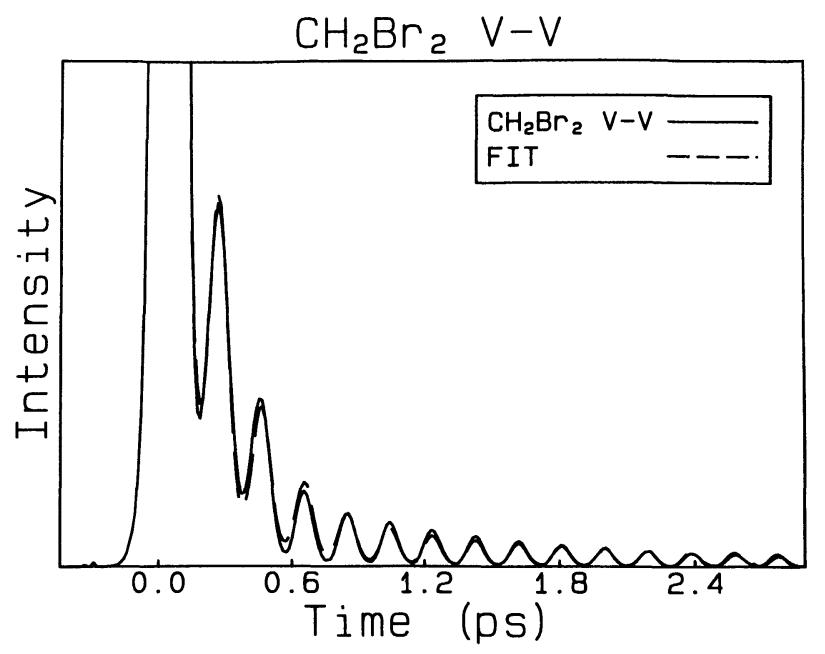

Fig. 11. - V-V ISRS data (solid curve) collected as shown schematically in figure 1 from dibromomethane liquid at room temperature. The oscillations in the data correspond to coherent vibrational oscillations of the $173-\mathrm{cm}^{-1}$ halogen bending mode in $\mathrm{CH}_{2} \mathrm{Br}_{2}$. The oscillations in signal decay due to molecular orientational motions and vibrational dephasing. The data also contain coherent Rayleigh-scattering contributions due to intermolecular and orientational motions. These effects were taken into account by equation (24) of the text, which was used to generate the fit (dashed curve). The fitting parameters are $\omega=33 \mathrm{ps}^{-1}, \quad \gamma=0.12 \mathrm{ps}^{-1}, \quad \Gamma_{1}=3.5 \mathrm{ps}^{-1}, \quad \Gamma_{2}=$ $0.30 \mathrm{ps}^{-1}$, and $A_{1}: A_{2}: B_{1}: B_{2}: C=3.7: 0: 13: 2.5: 1$.

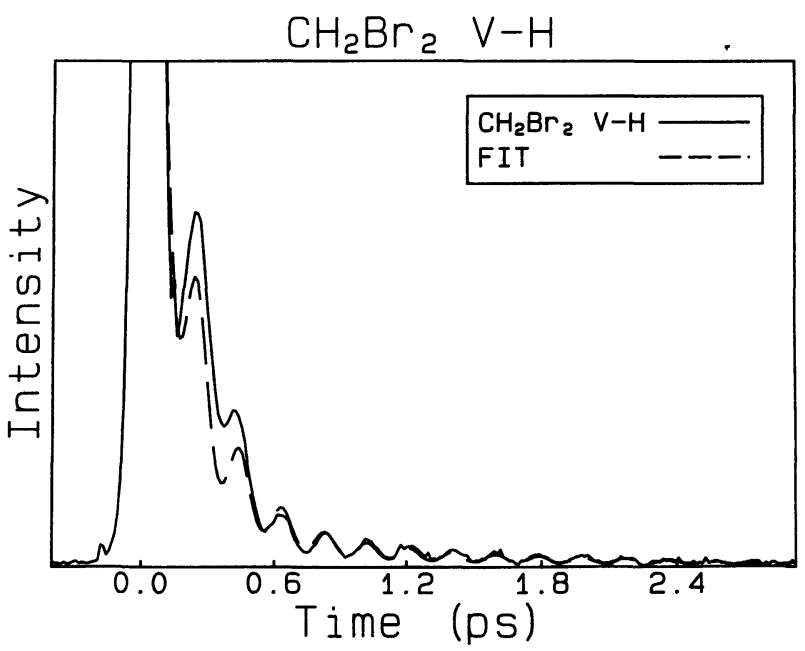

Fig. 12. - V-H ISRS data from $\mathrm{CH}_{2} \mathrm{Br}_{2}$ liquid (solid curve). The data contain the same contributions as in figure 11. The fit (dashed curve) was generated from equation (24) with the same dynamical parameters as in figure 11 and with

$$
A_{1}: A_{2}: B_{1}: B_{2}: C=2.1: 1: 16: 3.5: 0 \text {. }
$$

data in the first $200 \mathrm{fs}$, we approximate the Rayleigh contribution to $G^{\varepsilon \varepsilon}(t)$ (or to $C^{\varepsilon \varepsilon}(t)$ ) as a biexponential decay with «local » and collective relaxation rates $\Gamma_{1}$ and $\Gamma_{2}$, respectively. 
The amplitude of the oscillations in figures 11 and 12 decays rapidly during the first few oscillation periods, and then much more slowly. This is in accordance with frequency-domain results; see equation (14). Raman spectra of intramolecular vibrational modes in liquids typically show an approximately Lorentzian feature with a broad, nonLorentzian wing, qualitatively and often quantitatively similar to Rayleigh spectra but centred at the Stokes or anti-Stokes frequency shift rather than at zero frequency shift [1-6, 20, 27-34, 68]. The Lorentzian linewidth has contributions due to vibrational dephasing and also due to molecular orientational motion. The wing reflects faster components of orientational motion. Orientational motion contributes to time- or frequency-domain signal because the light-scattering differential polarizability for the molecular vibrational mode is a tensor quantity. In ISS, the excitation pulses drive vibrational motion most effectively on molecules which are preferentially aligned relative to the excitation polarization. Thus in V-V ISS, for example, molecules in some orientations (relative to $\mathrm{V}$ ) are vibrating with larger amplitudes than molecules in other orientations immediately following excitation. These same oriented molecules also contribute most to coherent scattering of the V-polarized probe pulse. The reorientational motions of the molecules will therefore reduce the coherent scattering intensity arising from vibrational motion [37]. For preliminary fitting of the data with a minimum number of adjustable parameters, we approximate the decay of oscillatory amplitude due to orientational motions as a biexponential decay with the same rates, $\Gamma_{1}$ and $\Gamma_{2}$, defined earlier. Essentially, we are assuming

$$
C_{i}^{\text {or }}(t) \sim A_{1} \mathrm{e}^{-\Gamma_{1} t}+A_{2} \mathrm{e}^{-\Gamma_{2} t},
$$

with a similar form for $C_{2}^{\text {Ray }}(t)$. We also assume that $C_{i}^{\text {vib }}(t) \sim \mathrm{e}^{-\gamma t} \cos \omega t$, with $\omega$ and $\gamma$ the vibrational frequency and dephasing rate, respectively.

From equation (11) and the above discussion, the ISRS signal due to molecular vibrational and orientational motions can be described approximately by the functional form,

$$
\begin{aligned}
I(t)=\left[\left(A_{1} \mathrm{e}^{-\Gamma_{1} t}+\right.\right. & \left.A_{2} \mathrm{e}^{-\Gamma_{2} t}+C\right) \mathrm{e}^{-\gamma t} \sin \omega t+ \\
& \left.+B_{1} \mathrm{e}^{-\Gamma_{1} t}+B_{2} \mathrm{e}^{-\Gamma_{2} t}\right]^{2},
\end{aligned}
$$

where $A_{1}, A_{2}, C, B_{1}$, and $B_{2}$ are amplitudes which depend on light-scattering cross-sections. $(C=0$ for $\mathrm{V}-\mathrm{H}$ ISRS). The data in figures 11 and 12 are fit reasonably well by equation (24) with the same dynamical parameters but with different amplitudes since the $\mathrm{V}-\mathrm{V}$ and $\mathrm{V}-\mathrm{H}$ scattering cross-sections differ.

Figure 13 shows ISRS data collected with an optical Kerr effect configuration. As discussed ear-

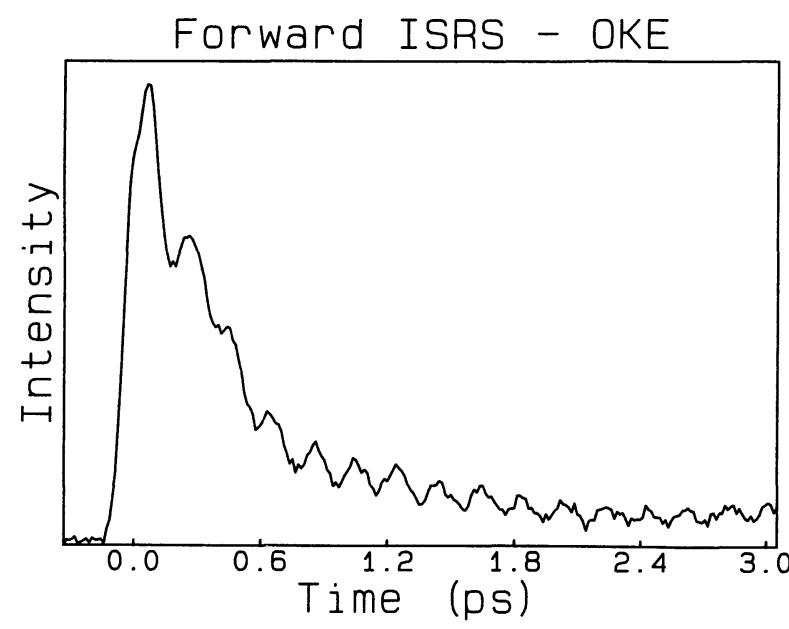

Fig. 13. - Forward-ISRS data from $\mathrm{CH}_{2} \mathrm{Br}_{2}$ liquid collected in a heterodyned optical Kerr effect configuration. The data contain the same contributions as in figures 11 and 12 .

lier, the results of OKE and V-H ISRS experiments in isotropic media are expected to be identical. In fact, the OKE data appear somewhat different because they were collected with a heterodyne signal also present due to slight depolarization of the probe pulse.

Figure 14 shows ISRS data collected with a single, V-polarized excitation pulse and a V-polarized, nearly collinear probe pulse. In this case, the transmitted probe pulse was dispersed by a monochromator and the intensities of frequency components on

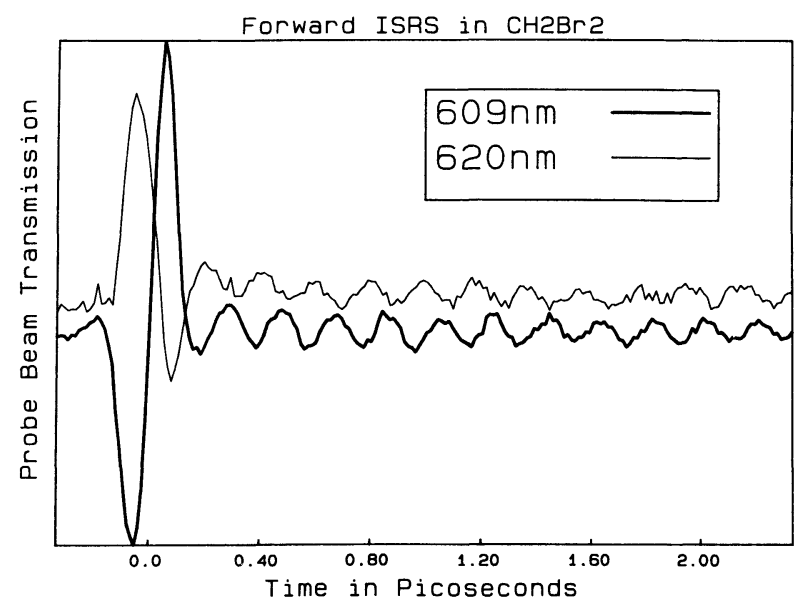

Fig. 14. - Forward-ISRS data from $\mathrm{CH}_{2} \mathrm{Br}_{2}$ liquid collected with a single, $\mathrm{V}$-polarized excitation pulse and a nearly collinear, V-polarized probe pulse. The spectrum of the incident probe pulse is centred at $615 \mathrm{~nm}$. Two frequency components of the transmitted probe pulse are monitored. The intensities of the red $(620-\mathrm{nm})$ and blue (609-nm) components undergo antiphased oscillations because the spectrum of the transmitted probe pulse is alternately red- and blue-shifted at the $\mathrm{CH}_{2} \mathrm{Br}_{2}$ molecular vibrational frequency. 
the blue side and the red side of the $615-\mathrm{nm}$ central frequency were monitored. Figure 14 shows that the intensities of the blue $(609 \mathrm{~nm})$ and red $(620 \mathrm{~nm})$ components undergo antiphased oscillations at the molecular vibrational frequency. This result is observed because, as discussed in section 2 , the spectrum of the transmitted probe «wags » back and forth between red and blue as the probe pulse arrives at the sample alternately in phase or out of phase with the coherent vibrational motion induced by the excitation pulse $[25,26,35]$.

These results illustrate some of the experimental configurations through which molecular vibrational motion may be time-resolved, and they confirm the theoretical expectations described in section 2 . Figures 15-17 present additional examples of $\mathrm{V}-\mathrm{V}$

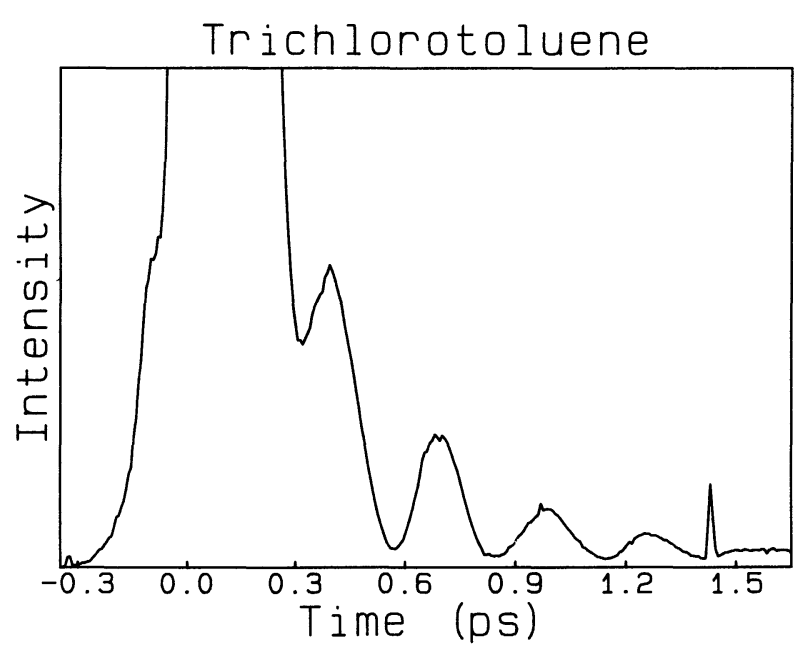

Fig. 15. - V-V ISRS data from trichlorotoluene liquid. The oscillations correspond to coherent vibrational oscillations of the $188-\mathrm{cm}^{-1}$ halogen bending mode of the molecule.

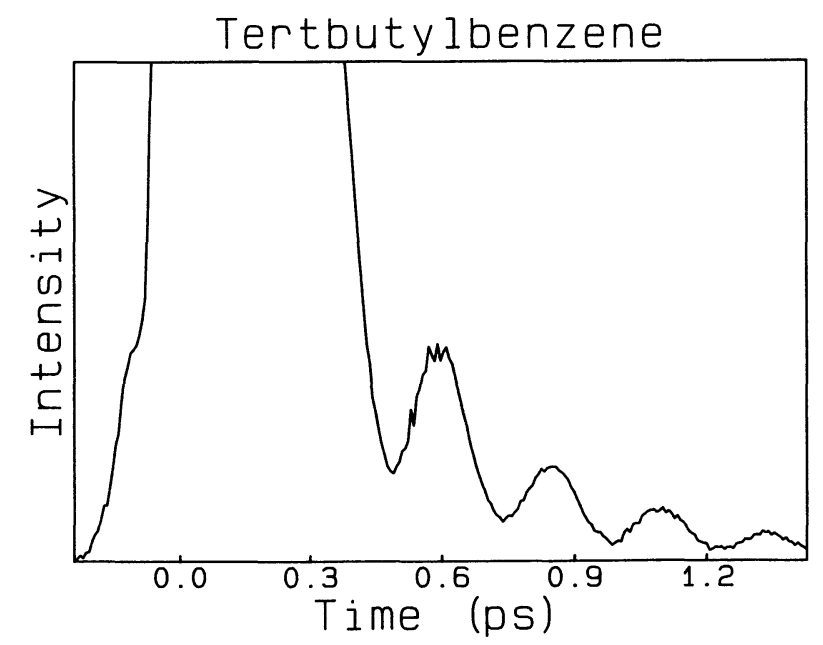

Fig. 16. $-\mathrm{V}-\mathrm{V}$ ISRS data from $t$-butylbenzene liquid, showing oscillations of the $135-\mathrm{cm}^{-1}$ methyl bending mode.

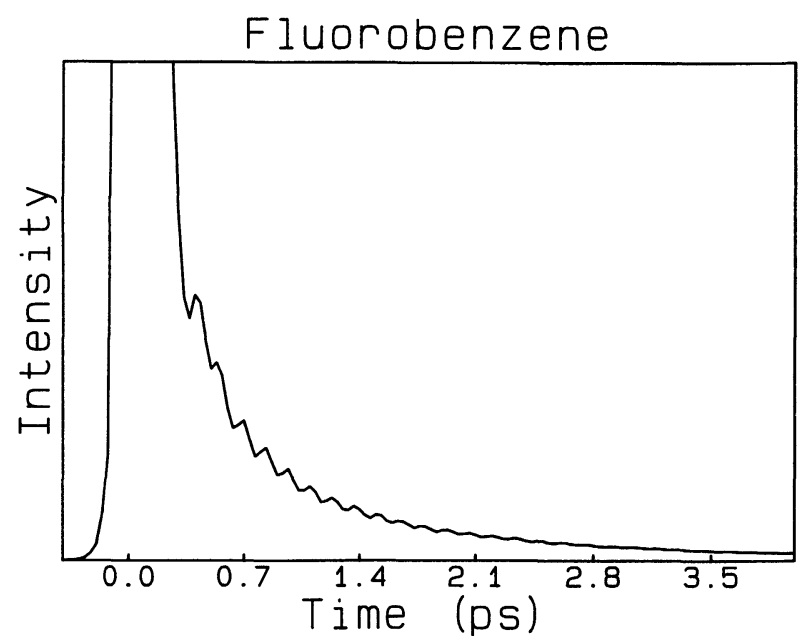

Fig. 17. - V-V ISRS data from fluorobenzene liquid. The oscillations are due to the $240-\mathrm{cm}^{-1}$ fluorene bending mode. The persistence of nonoscillatory signal shows that in this case the vibrational dephasing rate, $\gamma$, is more rapid than the orientational relaxation rate, $\Gamma_{2}$. (The data were taken with a $5 \times$ lower density of points than all the others and so appear less smooth.)

ISRS data collected from trichlorotoluene, $t$-butyl benzene, and fluorobenzene liquids at room temperature. The data all show temporal behaviour similar to that in figure 11 . In each case, the oscillation period in the data corresponds to the frequency of a Raman-active molecular vibrational mode [67]. Similar data from many other molecular liquids have been recorded. We note that the collection of such data in any of the experimental configurations described is extremely simple given stable, ultrashort pulses. In most of the V-V ISRS experiments, the coherently scattered signal and the oscillations in it were easily visible to the eye in a lit room, even with $<1 \mu \mathrm{J}$ total energy in the excitation pulses. This indicates that experiments on molecules in dilute solutions should be possible.

The results presented in this section demonstrate time-resolved initiation and observation of coherent molecular vibrational motion through ISRS. With the $60 \mathrm{fs}$ pulses used, vibrational modes of energies up to about $250 \mathrm{~cm}^{-1}$ could be time-resolved. These experiments complement frequency-domain Raman spectroscopy and may be useful to help extract information about orientational dynamics in the liquid phase [37, 70, 71]. Various types of « Raman echo » experiments involving sequences of excitation pulses are possible and may be of value in elucidating the various contributions to vibrational dephasing. Measurement of absolute light scattering cross-sections should also be straightforward.

These experiments open the door to many other kinds of spectroscopic observations that could be carried out on vibrationally distorted molecules. Of special interest will be time-resolved absorption 
spectroscopy [35]. The changes in electronic absorption spectra which are observed as molecules are stretched, compressed, or bent will directly yield vibronic interaction strengths and, if high-frequency vibrational progressions are affected, the coupling strengths between different vibrational modes.

\section{Concluding remarks.}

6.1 GENERAL OCCURRENCE OF ISS AND «IMPULSIVE » LIGHT-MATTER INTERACTIONS. - In this review we have shown theoretically and experimentally that ISRS can occur not only under the specialized excitation conditions of temporally and spatially overlapped pulses, but in fact with a single excitation pulse. ISRS must occur whenever a sufficiently short light pulse passes through any Ramanactive medium, resulting in coherent excitation of the medium and spectral changes in the pulse [35]. ISRS is therefore a general aspect of the way in which an ultrashort light pulse interacts with matter.

To generalize somewhat, we note that any lightmatter interaction which produces a resonant material excitation may become «impulsive » if the light pulse duration becomes short compared to the period of the material mode [8]. "Impulsive" absorption of ultrashort pulses has recently been demonstrated in several experiments [72-75]. In organic dye molecules, this resulted in coherent vibrational oscillation in excited electronic states [72-74] (unlike ISRS excitation which produces vibrational coherence in the ground electronic state). In electrooptic crystals, coherent polariton excitation was carried out by impulsive absorption of a far-IR pulse whose duration was only one optical cycle [75]. Each type of "impulsive » interaction has demon- strated interesting spectroscopic applications as well as effects which may be important in ultrafast optics.

6.2 Summary. - Femtosecond time-resolved impulsive stimulated scattering observations of intermolecular and intramolecular vibrational motions in molecular liquids have been presented and discussed. Intermolecular vibrations in $\mathrm{CS}_{2}$ and benzene liquids give rise to weakly oscillatory time-dependent ISS data. In $\mathrm{CS}_{2}$, the rapid loss of vibrational phase coherence appears to be due largely to inhomogeneity in the intermolecular librational frequency. Temperature-dependent trends in the librational frequency and dephasing dynamics have been elucidated.

ISRS experiments have permitted time-resolved observations of coherent intramolecular vibrations. These experiments should yield information about molecular vibrational and orientational dynamics, and will permit time-resolved spectroscopy of vibrationally distorted, nonequilibrium molecular structures.

\section{Acknowledgments.}

This work was supported in part by NSF Grants No. DMR-8306701 and DMR-8704352, U.S. ARO Contract No. DAAL03-86-K-0002, and NSF and private contributions to a Presidential Young Investigator Award. We gratefully acknowledge contributions from AT \& T Bell Laboratories, Monsanto, Chevron, DuPont, Perkin-Elmer, and TRW. We thank Prof. G. Mourou, T. Sizer, J. D. Kafka, and I. N. Duling for extensive advice on the construction of the femtosecond laser system, and Y.-X. Yan and Profs. I. Oppenheim and D. Kivelson for many helpful discussions.

\section{References}

[1] Molecular Liquids - Dynamics and Interactions, Eds A. J. Barnes, W. J. Orville-Thomas and J. Yarwood (R. Reidel) 1984.

[2] RothSCHILD, W. G., Dynamics of Molecular Liquids (Wiley, New York) 1984

[3] Coffey, W., Evans, M. and Grigolini, P., Molecular Diffusion and Spectra (Wiley, New York) 1984.

[4] Steele, W. A., Adv. Chem. Phys. 34 (1976) 1.

[5] Berne, B. J. and Pecora, R., Dynamic Light Scattering (John Wiley and Sons, New York) 1976.

[6] Kivelson, D. and Madden, P. A., Ann. Rev. Phys. Chem. 31 (1980) 523.

[7] Patterson, G. D. and Carroll, P. J., J. Phys. Chem. 89 (1985) 1344.

[8] A review of ISS theory and experiments is presented by Yan, Y.-X., Cheng, L.-T. and Nelson,
K. A., in Adv. Nonlinear Spectrosc. 16 Eds R. J. H. Clark and R. E. Hester (Wiley, Chichester) 1987 , p. 299 ; ISS experiments on viscoelastic liquids, in which dispersive acoustic behavior and structural relaxation dynamics were observed on ps-ns time scales, are not discussed in this review or the present paper. See YAN, Y.-X., ChenG, L.-T. and Nelson, K. A., J. Chem. Phys., in press.

[9] Robinson, M. M., Yan, Y.-X., GAMble, Jr., E. B., Williams, L. R., Meth, J. S. and Nelson, K. A., Chem. Phys. Lett. 112 (1984) 491 ;

FARrar, M. R., Cheng, L.-T., YAN, Y.-X. and NELSON, K. A., IEEE J. Quantum Electron. QE-22 (1986) 1453.

Cheng, L.-T. and Nelson, K. A., Phys. Rev. B, in press. 
[10] De Silvestri, S., Fujimoto, J. G., Ippen, E. P., Gamble, Jr. E. B., Williams, L. R. and Nelson, K. A., Chem. Phys. Lett. 116 (1985) 146.

[11] Williams, L. R., Ruhman, S., Joly, A. G., KoHLER, B. and NELSON, K. A., in Advances in Laser Science - II Eds M. Lapp, W. C. Stwalley and G. A. Kinney-Wallace (Proc. of 1986 ILS Conf., AIP, New York, 1987), and invited oral presentation at ILS Conf., Seattle, WA, October 1986.

[12] Ruhman, S., Williams, L. R., Joly, A. G., Kohler, B. and Nelson, K. A., J. Phys. Chem. 91 (1987) 2237.

[13] Ruhman, S., Kohler, B., Joly, A. G. and Nelson, K. A., IEEE J. Quantum Electron., in press.

[14] Ruhman, S., Kohler, B., Joly, A. G. and Nelson, K. A., Chem. Phys. Lett., in press.

[15] Kalpouzos, C., Lotshaw, W. T., McMorrow, D. and Kenney-Wallace, G. A., J. Phys. Chem. 91 (1987) 2028.

[16] Lotshaw, W. T., McMorrow, D., Kalpouzos, C. and Kenney-Wallace, G. A., Chem. Phys. Lett. 136 (1987) 323.

[17] Mctague, J. P. and Birnbaum, G., Phys. Rev. Lett. 21 (1968) 661.

[18] Madden, P. A., in Ultrafast Phenomena $I V$, Eds D. H. Auston and K. B. Eisenthal (SpringerVerlag, Berlin) 1984, p. 244.

[19] Madden, P. A. and Cox, T. I., Mol. Phys. 43 (1981) 287.

[20] Madden, P. A. and Tildesley, D. J., Mol. Phys. 55 (1985) 969.

[21] An, S. C., Fishman, L., Litovitz, T. A., MonTrose, C. J. and Posch, H. A., J. Chem. Phys. 70 (1979) 4626.

[22] Guillot, B., Bratos, S. and Birnbaum, G., Phys. Rev. A 22 (1980) 2230.

[23] Bucaro, J. A. and Litovitz, T. A., J. Chem. Phys. 54 (1971) 3846.

[24] Scarparo, M. A. F., Lee, J. H. and Song, J. I., Opt. Lett. 6 (1981) 193.

[25] Ruhman, S., Joly, A. G. and Nelson, K. A., J. Chem. Phys. 86 (1987) 6563.

[26] Ruhman, S., Joly, A. G. and Nelson, K. A., IEEE J. Quantum Electron., in press.

[27] Gordon, R. G., J. Chem. Phys. 42 (1965) $3658 ; 43$ (1965) 1307.

[28] Bartoli, F. J. and Litovitz, T. A., J. Chem. Phys. 56 (1972) 404 ; 413.

[29] Rosenthal, L. C. and Strauss, H. L., J. Chem. Phys. 64 (1976) 282.

[30] Cox, T. I., Battaglia, M. R. and Madden, P. A., Mol. Phys. 38 (1979) 1539.

[31] Schroeder, J., Schiemann, V. H. and Jonas, J., J. Chem. Phys. 69 (1978) 5479.

[32] Deb, S. K., Bansal, M. L. and Roy, A. P., Chem. Phys. 110 (1986) 391.

[33] Kluk, E., Zerda, T. W. and Zerda, J., Acta Phys. Pol. A 56 (1979) 121.

[34] Rothschild, W. G., J. Chem. Phys. 65 (1976) 455.

[35] Yan, Y.-X., Gamble, Jr. E. B. and Nelson, K. A., J. Chem. Phys. 83 (1985) 5391.
[36] Yan, Y.-X. and Nelson, K. A., J. Chem. Phys., in press.

[37] Time-resolved CARS and related techniques are reviewed in LAuberau, A. and Kaiser, W., Rev. Mod. Phys. 50 (1978) 607.

[38] Halbout, J.-M. and Tang, C. L., Appl. Phys. Lett. 40 (1982) 765.

[39] SHEN, Y. R., The Principles of Nonlinear Optics (John Wiley \& Sons, New York) 1984

[40] Sizer, T., KafKa, J. D., Duling, I. N., Gabel, C. W. and Mourou, G. A., IEEE J. Quantum Electron. QE-19 (1983) 506.

[41] Chesnoy, J. and FinI, L., Opt. Lett. 11 (1986) 635.

[42] Postlewaite, J. C., Miers, J. B., Reiner, C. C. and Dlott, D. D. et al., IEEE J. Quantum Electron., in press.

[43] Greene, B. I. and Farrow, R. C., J. Chem. Phys. 77 (1982) 4779.

[44] Halbout, J.-M. and Tang, C. L., Appl. Phys. Lett. 40 (1982) 765

[45] Greene, B. I. and Farrow, R. C., Chem. Phys. Lett. 98 (1983) 273.

[46] Greene, B. I., Fleury, P. A., Carter, Jr. H. L. and Farrow, R. C., Phys. Rev. A 29 (1984) 271.

[47] Hegemann, B., Baker, K. and Jonas, J., J. Chem. Phys. 80 (1984) 570 ;

Hegemann, B. and Jonas, J., J. Chem. Phys. 82 (1985) 2845.

[48] Enright, G. and Stoicheff, B. P., J. Chem. Phys. 60 (1974) 2536.

[49] Rothschild, W. G., Soussen-Jacob, J., Bessière, J. and Vincent-Geisse, J., J. Chem. Phys. 79 (1983) 3002.

[50] Lynden-Bell, R. J. and Steele, W. A., J. Phys. Chem. 88 (1984) 6514.

[51] Kabadi, V. N. and Steele, W. A., J. Phys. Chem. 89 (1985) 1467.

[52] Madden, P. and Kivelson, D., Adv. Chem. Phys. 16 (1984) 467.

[53] Rahman, A., J. Chem. Phys. 45 (1966) 2585.

[54] Ben-Reuven, A. and Zamir, E., J. Chem. Phys. 55 (1971) 475

[55] Kushick, J. N., J. Chem. Phys. 67 (1977) 2068.

[56] Steinhauser, O., Chem. Phys. Lett. 82 (1981) 153.

[57] Lynden-Bell, R. M., HutChinson, D. J. C. and Doyle, M. J., Mol. Phys. 58 (1986) 307.

[58] Cubeddu, R., Polloni, R., Sacchi, C. A. and Svelto, O., Phys. Rev. A 2 (1970) 1955.

[59] Hellwarth, R. W., Progr. Quantum Electron. 5 (1977) 1.

[60] Close, D. H., Giuliano, C. R., Hellwarth, R. W., Hess, L. D., MCClung, F. J. and WAGNER, W. G., IEEE J. Quantum Electron. QE-2 (1966) 553.

[61] We thank Prof. Erich Ippen for suggesting the V$\mathrm{V} / \mathrm{H}-\mathrm{H}$ experiment to reveal these effects.

[62] International Critical Tables, v. III, National Research Council of U.S.A. (McGraw-Hill, New York, 1928), p. 41.

[63] Keyes, T., Kivelson, D. and McTague, J. P., J. Chem. Phys. 55 (1971) 4096. 
[64] Ladanyi, B. M. and Keyes, T., J. Chem. Phys. 68 (1978) 3217.

[65] Frenkel, D. and Mctague, J. P., J. Chem. Phys. 72 (1980) 2801.

[66] Ladanyi, B. M., J. Chem. Phys. 78 (1983) 2189.

[67] Geiger, L. C. and Ladanyi, B. M., J. Chem. Phys. 87 (1987) 191.

[68] Wang, C. H., Jones, D. R. and Christensen, D. H., J. Chem. Phys. 64 (1976) 2820.

[69] Eds B. Schrader and W. Meier, DMS RAMAN/IR Atlas (Verlag Chemie GmbH, Weinheim) 1974 ; Sadtler Standard Raman Spectra (Sadtler, Philadelphia) 1974.
[70] Telle, H. R. and lauberau, A., Chem. Phys. Lett. 94 (1983) 467.

[71] Gale, G. M., Guyot-Sionnet, P. and Zheng, W. Q., Opt. Commun. 58 (1986) 395.

[72] Rosker, M. J., Wise, F. W. and Tang, C. L., Phys. Rev. Lett. 57 (1986) 321 ;

Wise, F. W., Rosker, M. J. and TANG, C. L., J. Chem. Phys. 86 (1987) 2827.

[73] Nelson, K. A. and Williams, L. R., Phys. Rev. Lett. 58 (1987) 745.

[74] HA, J. M. Y. et al., Phys. Rev. lett. 57 (1986) 3302.

[75] Cheung, K. P. and Auston, D. H., Phys. Rev. Lett. 55 (1985) 2152. 